\title{
Mixing and asymptotic distribution modulo 1
}

\author{
RUSSELL LYONS $\dagger$ \\ Department of Mathematics, Stanford University, Stanford CA 94305, USA
}

(Received 16 October 1986)

\begin{abstract}
If $\mu$ is a probability measure which is invariant and ergodic with respect to the transformation $x \mapsto q x$ on the circle $\mathbb{R} / \mathbb{Z}$, then according to the ergodic theorem, $\left\{q^{n} x\right\}$ has the asymptotic distribution $\mu$ for $\mu$-a.e. $x$. On the other hand, Weyl showed that when $\mu$ is Lebesgue measure, $\lambda$, and $\left\{\boldsymbol{m}_{j}\right\}$ is an arbitrary sequence of integers increasing strictly to $\infty$, the asymptotic distribution of $\left\{m_{j} x\right\}$ is $\lambda$ for $\lambda$-a.e. $x$. Here, we investigate the asymptotic distributions of $\left\{m_{j} x\right\} \mu$-a.e. for fairly arbitrary $\left\{\boldsymbol{m}_{j}\right\}$ under some strong mixing conditions on $\mu$. The result is a kind of stable ergodicity: the distributions are obtained from simple operations applied to $\mu$. The ideas extend to the situation of a sequence of transformations $x \mapsto q_{n} x$ where invariance is not present. This gives us information about many Riesz products and Bernoulli convolutions. Finally, we apply the theory to resolve some questions about $H$-sets.
\end{abstract}

\section{Introduction}

Suppose that $T$ is a continuous transformation on a compact metric space $X$. If $\mu$ is a $T$-invariant Borel probability measure on $X$, then the ergodic theorem says that for all $f \in L^{1}(\mu)$ and for $\mu$-a.e. $x$, the limit as $n \rightarrow \infty$ of

$$
\frac{1}{N} \sum_{n \leq N} f\left(T^{n} x\right)
$$

exists. If we restrict our attention to $f \in C(X)$, or a countable dense subset thereof, we see that $\left\{T^{n} x\right\}_{n \geq 1}$ has an asymptotic distribution, call it $\sigma_{x}$, for $\mu$-a.e. $x$ : that is, for $\mu$-a.e. $x$,

$$
\frac{1}{N} \sum_{n \leq N} f\left(T^{n} x\right) \rightarrow \int_{X} f d \sigma_{x} \quad \text { for all } f \in C(X) .
$$

We write $\left\{T^{n} x\right\} \sim \sigma_{x} \mu$-a.e. Evidently, $\sigma_{x}$ is $T$-invariant and integrating (1) with respect to $\mu$ shows that

$$
\mu=\int_{X} \sigma_{x} d \mu(x)
$$

in the weak sense. The measure $\mu$ is ergodic if and only if $\sigma_{x}=\mu$ a.e. The Bogoliouboff theory [12] shows that in any case $\sigma_{x}$ is ergodic $\mu$-a.e. The integral (2) is thus a convex combination of invariant measures in terms of ergodic measures (the extreme points).

† Partially supported by an AMS Research Fellowship. 
We are interested in the case where $T$ is the transformation $T_{q}: x \mapsto q x$ on the circle $\mathbb{T}=\mathbb{R} / \mathbb{Z}, q \in \mathbb{Z},|q| \geq 2$. The additional structure on the circle interacts with ergodic theory in many interesting ways. We intend to explore here the asymptotic distribution of $\left\{m_{j} x\right\}$ for sequences other than simply $m_{j}=q^{j}$. For example, Weyl $[17, \S 7]$ showed that when $\mu$ is Lebesgue measure, $\lambda$, and $\left\{m_{j}\right\}$ is an arbitrary sequence of integers increasing strictly to $\infty$, then $\left\{m_{j} x\right\} \sim \lambda$ for $\lambda$-a.e. $x$. Now given an arbitrary sequence $\left\{\boldsymbol{m}_{j}\right\}$ and measure $\mu,\left\{m_{j} x\right\}$ need not possess an asymptotic distribution on a set of non-zero $\mu$-measure. However, there always does exist [8] a subsequence $\left\{\boldsymbol{m}_{j}^{\prime}\right\}$ of $\left\{\boldsymbol{m}_{j}\right\}$, which we denote simply by $\left\{\boldsymbol{m}_{j}^{\prime}\right\} \subset\left\{\boldsymbol{m}_{j}\right\}$, such that even for any further subsequence $\left\{m_{j}^{\prime \prime}\right\} \subset\left\{m_{j}^{\prime}\right\}$ and for $\mu$-a.e. $x$, the sequence $\left\{m_{j}^{\prime \prime} x\right\}$ has an asymptotic distribution $\sigma_{x}$. Hence we shall restrict our attention to sequences $\left\{m_{j}\right\}$ already enjoying this property of stability: that is, we assume that there exists $\sigma: \mathbb{T} \rightarrow M(\mathbb{T})$ such that for all $\left\{m_{j}^{\prime}\right\} \subset\left\{m_{j}\right\}$ and for $\mu$-a.e. $x\left\{m_{j}^{\prime} x\right\} \sim \sigma_{x}$. We remark that if $\left\{q^{j}\right\}$ itself is to have this property for a $q$-invariant $\mu$, then it is necessary (though not sufficient) that $\mu$ be $q$-mixing:

$$
\forall f, g \in L^{2}(\mu) \int f\left(T_{q}^{n} x\right) g(x) d \mu(x) \rightarrow\left(\int f d \mu\right)\left(\int g d \mu\right),
$$

or, equivalently,

$$
\forall a, b \in \mathbb{Z} \hat{\mu}\left(a q^{n}+b\right) \rightarrow \hat{\mu}(a) \hat{\mu}(b)
$$

where

$$
\hat{\mu}(k)=\int_{\pi} e(-k x) d \mu(x), e(x)=e^{2 \pi i x} .
$$

We shall impose an even stronger mixing condition on $\mu$ in order to determine $\sigma_{x}$ for any (stable) $\left\{\boldsymbol{m}_{j}\right\}$. Now $\sigma_{x}$ is determined by its Fourier-Stieltjes coefficients, $\hat{\sigma}_{x}(r), r \in \mathbb{Z}$. By (1),

$$
\frac{1}{J} \sum_{j \leq J} e\left(-r m_{j}^{\prime} x\right) \rightarrow \hat{\sigma}_{x}(r) \quad \mu \text {-a.e. }
$$

for all $\left\{m_{j}^{\prime}\right\} \subset\left\{m_{j}\right\}$, whence

$$
\forall r \in \mathbb{Z} e\left(-r m_{j} x\right) \rightarrow \hat{\sigma}_{x}(r) \text { weak }^{*} \text { in } L^{\infty}(\mu) .
$$

(Here, we regard $L^{\infty}(\mu)$ as the dual of $L^{1}(\mu)$.) The problem is thus equivalent to determining the simultaneous weak* limits of $e\left(-r m_{j} x\right)$ in $L^{\infty}(\mu)$.

If we integrate (3) and let $\Sigma$ be the measure such that $\hat{\Sigma}(r)=\lim _{j \rightarrow \infty} \hat{\mu}\left(-r m_{j} x\right)$, then we obtain a formula analogous to (2):

$$
\Sigma=\int_{\top} \sigma_{x} d \mu(x)
$$

Another way of viewing (3) and (3a) is given in [10].

The reader may find it easier to follow our proofs if he first works out the following set of examples, in which the most important phenomena are present. We take $q=3$ and $\mu$ the Riesz product [5, p. 107]

$$
\mu=\prod_{k \geq 0}\left(1+\operatorname{Re}\left\{\alpha e\left(3^{k} x\right)\right\}\right)
$$


where $|\alpha| \leq 1$. If $m_{j}=3^{j}$, then $\sigma_{x}=\mu$ a.e. If $m_{j}=3^{2 j}+3^{j}$, then $\sigma_{x}=\mu * \mu=\mu^{2}$ a.e. If $m_{j}=3^{j}+1$, then $\sigma_{x}=\mu * \delta(x)$ a.e., where $\delta(x)$ is the unit mass at $x$. If $m_{j}=2 \cdot 3^{j}$, then $\sigma_{x}$ is the measure such that $\hat{\sigma}_{x}(r)=\hat{\mu}(2 r)$ a.e. If

$$
m_{j}=\frac{3^{j}-1}{2}=3^{j-1}+3^{j-2}+\cdots+3+1
$$

then $\sigma_{x}$ is the measure such that

$$
\hat{\sigma}_{x}(r)= \begin{cases}\hat{\omega}(r / 2) & \text { if } r \text { is even, } \\ 0 & \text { if } r \text { is odd }\end{cases}
$$

where $\omega=\mu * \delta(-x)$.

\section{The invariant case}

For any integer $q$, we let $T_{q}$ be the operator on $M(\mathbb{T})$ such that

$$
\left(T_{q} \omega\right) \hat{(}(n)=\hat{\omega}(q n) \quad(n \in \mathbb{Z}, \omega \in M(\mathbb{T})) .
$$

If $q \neq 0$, we define $T_{q}^{-1}$ by

$$
\left(T_{q}^{-1} \omega\right)^{\wedge}(n)= \begin{cases}\hat{\omega}(n / q) & \text { if } q \mid n, \\ 0 & \text { if } q \nmid n,\end{cases}
$$

while if $q=0$, we set $T_{0}^{-1} \omega=\hat{\omega}(0) \lambda$, where $\lambda$ is Lebesgue measure. Thus for $q \neq 0$, $T_{q} \circ T_{q}^{-1}=\mathrm{id}$. It is easily checked that

$$
\omega * T_{q}^{-1} \omega^{\prime}=T_{q}^{-1}\left[T_{q} \omega * \omega^{\prime}\right] .
$$

The hypotheses of our first theorem below are immediately seen to be satisfied by Riesz products,

$$
\mu=\prod_{k \geq 0}\left(1+\operatorname{Re}\left\{\alpha e\left(q^{k} x\right)\right\}\right), \quad|\alpha| \leq 1, \quad|q| \geq 3,
$$

and it is not difficult to verify them for Bernoulli convolutions $([4$, p. 182])

$$
\mu=\underset{k \geq 1}{*}\left(p \delta(0)+(1-p) \delta\left(2^{-k}\right)\right), \quad 0<p<1
$$

(here, $q=2$ ), for example. After the proof of the theorem, we shall discuss the hypotheses more thoroughly, including their mixing character.

THEOREM 1. Let $\mu$ be a q-invariant probability measure such that

$$
\forall b \in \mathbb{Z} \lim _{n \rightarrow \infty} \sup _{a \in \mathbb{Z}}\left|\hat{\mu}\left(a q^{n}+b\right)-\hat{\mu}(a) \hat{\mu}(b)\right|=0
$$

and

given any sequence $\left\{e\left(m_{j} x\right)\right\}_{j \geq 1}$ which does not converge to 0 weak ${ }^{*}$ in $L^{\infty}(\mu)$, there exists a subsequence $\left\{m_{j}^{\prime}\right\} \subset\left\{m_{j}\right\}$ and integers $b, a_{j}, n_{j}$ such that $n_{j} \rightarrow \infty$ and

$$
m_{j}^{\prime}=a_{j} q^{n_{j}}+b
$$

Then if $\left|m_{j}\right| \rightarrow \infty$ is such that (3) holds, there exist integers $r, b$, an integer $l \geq 1$, and non-zero integers $s_{1}, \ldots, s_{l}$ such that

$$
\sigma_{x}=T_{r}^{-1}\left[\delta(b x) * T_{s_{1}} \mu * \cdots * T_{s_{1}} \mu\right] \quad \mu \text {-a.e. }
$$


We shall find it handy to use the equivalence of $(6)$ to

$$
\text { if } \lim _{n \rightarrow \infty} \lim _{j \rightarrow \infty} d_{n}\left(m_{j}\right)=\infty \text {, then } e\left(m_{j} x\right) \rightarrow 0 \text { weak* in } L^{\infty}(\mu),
$$

where $d_{n}(m)=\left|m-q^{n} \mathbb{Z}\right|$, i.e., the distance from $m$ to the multiples of $q^{n}$. It is straightforward to show that (6) implies (8). Conversely, suppose that (8) holds and let $e\left(m_{j} x\right) \nrightarrow 0$ weak* in $L^{\infty}(\mu)$. Then by compactness, there is a subsequence $\left\{e\left(m_{j}^{\prime \prime} x\right)\right\}$ having a non-zero weak* limit. Let $\left\{m_{j}^{\prime \prime \prime}\right\} \subset\left\{m_{j}^{\prime \prime}\right\}$ be such that for all $n \geq 1$, $\lim _{j \rightarrow \infty} d_{n}\left(m_{j}^{\prime \prime \prime}\right)$ exists. Then $e\left(m_{j}^{\prime \prime \prime} x\right) \nrightarrow 0$ and by $(8)$, since $\lim _{j \rightarrow \infty} d_{n}\left(m_{j}^{\prime \prime \prime}\right)$ is increasing in $n$, for large enough $n$, say $n>N$, we have $\lim _{j \rightarrow \infty} d_{n}\left(m_{j}^{\prime \prime \prime}\right)=\tilde{b}<\infty$. This means that for $n>N$ and for $j \geq J(n)$, we can write $m_{j}^{\prime \prime \prime}=\tilde{a}_{j} q^{n}+b$, where $b= \pm \tilde{b}$ and is fixed without loss of generality. In particular, $m_{J(n)}^{\prime \prime \prime}=\tilde{a}_{J(n)} q^{n}+b$ for $n>N$. Thus, $m_{k}^{\prime}=m_{J(N+k)}^{\prime \prime \prime}$ defines the required sequence.

Proof. Let $\left|m_{j}\right| \rightarrow \infty$ be such that (3) holds. If $\sigma_{x}=\lambda \mu$-a.e., then we take $r=0$ in (7) and we are done. In the contrary case, there is an $r \neq 0$ such that $\hat{\sigma}_{x}(r) \neq 0$. By a diagonal procedure, we may find a subsequence $\left\{m_{j}^{\prime}\right\}$ - which we shall take to be the whole sequence without loss of generality - such that for all $n \geq 1$ and all $r$, $\lim _{j \rightarrow \infty} d_{n}\left(r m_{j}\right)$ exists. Thus by $(8)$, the set

$$
E=\left\{r: \lim _{n \rightarrow \infty} \lim _{j \rightarrow \infty} d_{n}\left(r m_{j}\right)<\infty\right\}
$$

is not just $\{0\}$. We claim that $E=r_{0} \mathbb{Z}$ for some $r_{0}>0$. It suffices to show that $E$ is a subgroup of $\mathbb{Z}$. But if $r, s \in E$, then $d_{n}\left((r-s) m_{j}\right) \leq d_{n}\left(r m_{j}\right)+d_{n}\left(s m_{j}\right)$, whence $r-s \in E$ and so $E$ is indeed a subgroup. It follows that if $r$ is not a multiple of $r_{0}$, then $\hat{\sigma}_{x}(r)=0 \mu$-a.e., whence there exists $\nu_{x}$ such that $\sigma_{x}=T_{r_{0}}^{-1} \nu_{x} \mu$-a.e.

We must now determine $\nu_{x}$. Since $r_{0} \in E$, we can, by replacing $\left\{m_{j}\right\}$ by a subsequence if necessary, suppose that there are integers $a_{j}, n_{j}, b_{0}$ such that $r_{0} m_{j}=$ $a_{j} q^{n_{j}}+b_{0}, n_{j} \rightarrow \infty, q \nmid a_{j}$, and also that $\left\{e\left(r a_{j} x\right)\right\}_{j}$ has a weak ${ }^{*}$ limit in $L^{\infty}(\mu)$ for each $r$. Let $\hat{\Sigma}_{1}(r)=\lim _{j \rightarrow \infty} \hat{\mu}\left(r a_{j}\right)$. We claim that

$$
\forall r \in \mathbb{Z} \hat{\nu}_{x}(r)=\hat{\Sigma}_{1}(r) e\left(-r b_{0} x\right) \quad \mu \text {-a.e. }
$$

Indeed, for all $s$, we have

$$
\begin{aligned}
\int_{\mathrm{T}} \hat{\nu}_{x}(r) e(-s x) d \mu(x)=\int \hat{\sigma}_{x}\left(r r_{0}\right) e(-s x) d \mu(x) \\
=\lim _{j \rightarrow \infty} \int e\left(-r r_{0} m_{j} x\right) e(-s x) d \mu(x)=\lim _{j \rightarrow \infty} \hat{\mu}\left(r r_{0} m_{j}+s\right) \\
=\hat{\Sigma}_{1}(r) \hat{\mu}\left(r b_{0}+s\right) \quad(\text { by }(5)) \\
=\int \hat{\Sigma}_{1}(r) e\left(-r b_{0} x\right) e(-s x) d \mu(x) .
\end{aligned}
$$

From (9), it follows that $\nu_{x}=\delta\left(b_{0} x\right) * \Sigma_{1} \mu$-a.e. and it remains to determine $\Sigma_{1}$.

Since $\Sigma_{1} \neq \lambda$ (otherwise, $\sigma_{x}=\lambda$ a.e.), we can argue as in the first paragraph to obtain $r_{1}>0$ such that $\Sigma_{1}=T_{r_{1}}^{-1} \Sigma_{1}^{\prime}$, where, taking a subsequence of $\left\{a_{j}\right\}$ if necessary as in the second paragraph, we can assume that

$$
r_{1} a_{j}=a_{j}^{(2)} q^{n_{j}^{\prime}}+s_{1}^{\prime}, n_{j}^{\prime} \rightarrow \infty, \quad q \nmid a_{j}^{(2)} \text { or } a_{j}^{(2)}=0,
$$


and $\hat{\Sigma}_{1}^{\prime}(r)=\hat{\mu}\left(r s_{1}^{\prime}\right) \hat{\Sigma}_{2}(r)$, where $\hat{\Sigma}_{2}(r)=\lim _{j \rightarrow \infty} \hat{\mu}\left(r a_{j}^{(2)}\right)$. Thus, $\Sigma_{1}=T_{r_{1}}^{-1}\left[T_{s i} \mu * \Sigma_{2}\right]$. Note that $s_{1}^{\prime} \neq 0$. We then proceed for $\Sigma_{2}$ as we did for $\Sigma_{1}$, and so on. This process ends if and only if $a_{j}^{(l+1)} \equiv 0$ for some $l$. We claim the process must indeed end. Otherwise, for each $r,\left|\hat{\sigma}_{x}(r)\right|$ would be bounded by $\left[\sup _{n \neq 0}|\hat{\mu}(n)|\right]^{l}$ for each $l$; since $\sigma_{x} \neq \lambda$, it follows that $\sup _{n \neq 0}|\hat{\mu}(n)|=1$. Thus there exists $\left\{N_{j}\right\}$ such that $\left|\hat{\mu}\left(N_{j}\right)\right| \rightarrow 1$. By (6), (5), and $q$-invariance, there is then a $b \neq 0$ such that $|\hat{\mu}(b)|=1$. This, of course, implies that $\mu$ has finite support and that $\hat{\mu}$ is periodic, which contradicts (6).

We have thus obtained the expression

$$
\sigma_{x}=T_{r_{0}}^{-1}\left[\delta\left(b_{0} x\right) * T_{r_{1}}^{-1}\left[T_{s_{1}} \mu * T_{r_{2}}^{-1}\left[T_{s_{2}} \mu * \cdots * T_{r_{1}}^{-1} T_{s i} \mu\right]\right] \ldots\right] \mu \text {-a.e. }
$$

Use of (4) $l$ times yields (7) with $r=r_{0} r_{1} \cdots r_{l}, b=b_{0} r_{1} \cdots r_{l}, s_{i}=s_{i}^{\prime} r_{i+1} r_{i+2} \cdots r_{l}$ for $1 \leq i<l$, and $s_{l}=s_{l}^{\prime}$.

Recall that if $T$ is a measure-preserving transformation of a Lebesgue space $(X, \mathscr{B}, \mu)$, then $T$ (or $\mu$ ) is called exact if the $\sigma$-field

$$
\text { Tail }(\mathscr{B}) \stackrel{\text { def }}{=} \bigcap_{n \geq 0} T^{-n_{\mathscr{B}}}
$$

is trivial, i.e. consists only of sets of measure 0 or 1 [3, p. 289]. (This is the same as saying that Kolmogorov's $0-1$ law holds.) There are several convenient equivalent conditions which depend on the following notions. If $\xi$ is a partition of $X$, let $\mathscr{B}(\xi)$ denote the smallest complete sub- $\sigma$-field of $\mathscr{B}$ containing those measurable sets which are unions of elements of $\xi$. We say that $\xi$ is trivial if $\mathscr{B}(\xi)$ is trivial. Let Tail $(\xi)$ denote the partition $\bigwedge_{n \geq 0} \vee_{k \geq n} T^{-k} \xi$. For a set $A$, let Tail $(A)=$ $\bigcup_{n \geq 0} T^{-n} T^{n} A$. Thus, Tail $(\mathscr{B})=\{$ Tail $(A): A \in \mathscr{B}\}$. It is not hard to demonstrate that the following conditions are equivalent (see [3, pp. 283-4], [15], or [16, Chap. VII]):

(i) $T$ is exact;

(ii) for any finite partition $\xi$, Tail $(\xi)$ is trivial;

(iii) for every set $A$ of positive measure, $\mu$ (Tail $(A))=1$;

(iv) if $\langle f, g\rangle$ denotes $\int f \bar{g} d \mu$, then

$$
\forall g \in L^{2}(\mu) \lim _{n \rightarrow \infty} \sup _{\substack{f \in L^{2}(\mu) \\\|f\|_{2} \leq 1}}\left|\left\langle T^{n} f, g\right\rangle-\langle f, 1\rangle\langle 1, g\rangle\right|=0 ;
$$

(v) $T$ is $K$-mixing, i.e. if $\xi$ is any finite partition, then

$$
\forall r \geqslant 1 \quad \forall B \in \bigvee_{k \leq r} T^{-k} \xi \lim _{n \rightarrow \infty} \sup _{A \in \bigvee_{k \geq n} T^{-k} \xi}|\mu(A \cap B)-\mu(A) \mu(B)|=0 .
$$

Furthermore, if $\xi$ is some finite generating partition (i.e., $\mathscr{B}\left(\bigvee_{n \geq 0} T^{-n} \xi\right)=\mathscr{B}$ ) and Tail $(\xi)$ is trivial or (11) holds, then $T$ is exact.

In our case, the partition

$$
\xi_{q}= \begin{cases}\left\{\left[\frac{i}{q}, \frac{i+1}{q}[: 0 \leq i<q\}\right.\right. & \text { if } q>0, \\ \left\{\left[\frac{1}{|q|+1}+\frac{i}{|q|}, \frac{1}{|q|+1}+\frac{i+1}{|q|}[: 0 \leq i<|q|\}\right.\right. & \text { if } q<0\end{cases}
$$


is generating for $T=T_{q}$ since $\bigvee_{n \geq 0} T_{q}^{-n} \xi_{q}$ is the discrete partition $\{\{x\}: x \in \mathbb{T}\}$. Evidently, our hypothesis (5) is a bit weaker than (10), i.e., than exactness. By using the partition $\xi_{q}$ and (11), we immediately deduce the exactness of any Bernoulli convolution

$$
\mu=\underset{k \geq 1}{*}\left[p_{0} \delta(0)+p_{1} \delta\left(q^{-k}\right)+\cdots+p_{|q|-1} \delta\left((|q|-1) q^{-k}\right)\right],
$$

where $0 \leq p_{i} \leq 1, p_{0}+p_{1}+\cdots+p_{|q|-1}=1$, and $p_{i} \neq 0$ for at least two $i$ 's. (Note that when $q<0$,

$$
\begin{aligned}
\mu & =\delta\left(\frac{1}{|q|+1}\right) * \mu *\left(\underset{k \text { odd }}{*} \delta\left((|q|-1)|q|^{-k}\right)\right) \\
& =\delta\left(\frac{1}{|q|+1}\right) *\left[\underset{\substack{* \\
k \text { even }}}{\mid} \sum_{i=0}^{|q|-1} p_{i} \delta\left(i|q|^{-k}\right)\right] *\left[\underset{\substack{* \\
k \text { odd }}}{*} \sum_{i=0}^{|q|-1} p_{|q|-1-i} \delta\left(i|q|^{-k}\right)\right] .
\end{aligned}
$$

Also, $\left(\mathbb{T}, \mu, T_{q}\right)$ is metrically isomorphic to $\left(\mathbb{T}, \nu, T_{|q|}\right)$ via the mapping

$$
\sum_{k \geq 1} \varepsilon_{k} q^{k} \mapsto \sum_{k \geq 1} \varepsilon_{k}|q|^{-k}
$$

$\left(0 \leq \varepsilon_{k}<|q|\right)$, where $\nu=*_{k \geq 1} \sum_{i=0}^{|q|-1} p_{i} \delta\left(i|q|^{-k}\right)$.) We remark that an approximation argument quickly shows that Riesz products satisfy (10) as well. A stronger result $[11,13]$ is that Riesz products are isomorphic to Bernoulli shifts.

The hypothesis (5) is also equivalent to the following kind of tameness [4, Chapter 6] of $\mu$ : if $e\left(a_{n} q^{n} x\right) \rightarrow \chi(x)$ weak* in $L^{\infty}(\mu)$, then $\chi$ is constant a.e. We leave this as an exercise.

We now turn to the hypothesis (6). Rather than being of a purely mixing character, it links an arbitrary sequence $\left\{m_{j}\right\}$ to the transformation $T_{q}$. It too can be thought of as a tameness condition, for if $\mu$ satisfies (5) and (6), then $\mu$ is 'weakly tame': if $e\left(m_{j} x\right) \rightarrow \chi(x)$ weak $^{*}$ in $L^{\infty}(\mu)$, then $\chi(x)=c e(n x)$ a.e. for some constant $c$ and some integer $n$. In any case, it is obvious that Riesz products satisfy (6) and this is not hard to see for Bernoulli convolutions (12) with $p_{0} p_{1} \neq 0$. Indeed, we shall establish (6) assuming that $\operatorname{gcd}\left\{i-i_{0}: p_{i} \neq 0\right\}=1$, where $i_{0}$ is any subscript such that $p_{i_{0}} \neq 0$. First note that if $e\left(m_{j} x\right) \nrightarrow^{w *} 0$ in $L^{\infty}(\mu)$, then for some $m$,

$$
\hat{\mu}\left(m_{j}+m\right)=\int e\left(-m_{j} x\right) e(-m x) d \mu(x) \nrightarrow 0 .
$$

By replacing the sequence $\left\{m_{j}\right\}$ with $\left\{m_{j}+m\right\}$, we may assume that $m=0$. Now if $\hat{\mu}\left(m_{j}\right) \nrightarrow 0$, then

$$
\prod_{k \geq 1}\left(\sum_{i} p_{i} e\left(-i m_{j} q^{-k}\right)\right) \nrightarrow 0 \quad \text { as } j \rightarrow \infty .
$$

By taking a subsequence if necessary, we may assume that for all $i$ and $k$, $\lim _{j \rightarrow \infty} e\left(-i m_{j} q^{-k}\right)$ and $\lim _{j \rightarrow \infty} \hat{\mu}\left(m_{j}\right)$ exist. It follows that

$$
\infty>\lim _{j \rightarrow \infty} \sum_{k \geq 1}\left[1-\left|\sum_{i} p_{i} e\left(-i m_{j} q^{-k}\right)\right|\right] \geq \sum_{k \geq 1}\left[1-\left|\sum_{i} p_{i} \lim _{j \rightarrow \infty} e\left(-i m_{j} q^{-k}\right)\right|\right],
$$

so that for some $\theta_{k}$,

$$
\lim _{k \rightarrow \infty} e\left(\theta_{k}\right) \sum_{i} p_{i} \lim _{j \rightarrow \infty} e\left(-i m_{j} q^{-k}\right)=1
$$


Thus, for $p_{i} \neq 0$,

$$
\lim _{k \rightarrow \infty} e\left(\theta_{k}\right) \lim _{j \rightarrow \infty} e\left(-i m_{j} q^{-k}\right)=1 .
$$

If $p_{i_{0}} \neq 0$, we then have $\lim _{k \rightarrow \infty} \lim _{j \rightarrow \infty} e\left(-\left(i-i_{0}\right) m_{j} q^{-k}\right)=1$, so that the hypothesis $\operatorname{gcd}\left\{i-i_{0}: p_{i} \neq 0\right\}=1$ implies that $\lim _{k \rightarrow \infty} \lim _{j \rightarrow \infty} e\left(-m_{j} q^{-k}\right)=1$, which is the same as $\lim _{k} \lim _{j}\left\|m_{j} q^{-k}\right\|=0$, where $\|x\|=|x-\mathbb{Z}|$. We have only to apply the following lemma to be able to conclude (8) and thus (6). (More precise information on sequences $\left\{m_{j}\right\}$ such that $\hat{\mu}\left(m_{j}\right) \rightarrow 0$ is given in [2] for certain $\mu$.)

LEMMA 2. $\lim _{k \rightarrow \infty} \overline{\lim }_{j \rightarrow \infty} d_{k}\left(m_{j}\right)=\infty \Leftrightarrow \overline{\lim }_{k \rightarrow \infty} \overline{\lim }_{j \rightarrow \infty}\left\|m_{j} q^{-k}\right\|>0$.

Proof. Since $\left\|m_{j} q^{-k}\right\|=|q|^{-k} d_{k}\left(m_{j}\right)$, the implication $(\Leftarrow)$ is immediate. Conversely, suppose that $\lim _{k} \varlimsup_{\lim _{j}} d_{k}\left(m_{j}\right)=\infty$. Then for all $N$ there is a $k=k(N)$ such that $\varlimsup_{j} d_{k}\left(m_{j}\right) \geq N$. Let $\left\{j_{l}\right\}$ be a sequence such that for all $l, d_{k}\left(m_{j_{i}}\right)=N_{1} \geq N$. Let $n=n(N)$ be the least integer such that $|q|^{n} / 2 \geq N_{1}$. Then $k \geq n$ and for all $l$, $d_{n}\left(m_{j_{l}}\right)=d_{k}\left(m_{j_{l}}\right)=N_{1}$ and $\left\|m_{j,} q^{-n}\right\|=|q|^{-n} d_{n}\left(m_{j_{l}}\right)=|q|^{-n} N_{1}>1 /|2 q|$. Since $n(N) \rightarrow$ $\infty$ as $N \rightarrow \infty$, it follows that $\varlimsup_{\lim _{k}} \varlimsup_{\lim _{l}}\left\|m_{j l} q^{-k}\right\| \geq 1 /|2 q|$.

Suppose, on the other hand, that the Bernoulli convolution (12) satisfies $\operatorname{gcd}\left\{i-i_{0}: p_{i} \neq 0\right\}=r_{0} \neq 1, p_{i_{0}} \neq 0$. We may assume that $i_{0}=\min \left\{i: p_{i} \neq 0\right\}$. Consider the measure

$$
\begin{aligned}
\mu * \delta\left(-\frac{i_{0}}{q-1}\right) & =\underset{k \geq 1}{*}\left[\left(\sum_{i} p_{i} \delta\left(i q^{-k}\right)\right) * \delta\left(-i_{0} q^{-k}\right)\right] \\
& =\underset{k \geq 1}{*}\left(\sum_{i} p_{i} \delta\left(\left(i-i_{0}\right) q^{-k}\right)\right)=T_{r_{0}} \nu,
\end{aligned}
$$

where

$$
\nu=\underset{k \geqslant 1}{*} \sum_{i} p_{i} \delta\left(\frac{i-i_{0}}{r_{0}} q^{-k}\right)
$$

By definition of $r_{0}$ and what we've just proved, $\nu$ satisfies (6) and, of course, (5). Since Theorem 1 applies to $\nu$, we claim that if (3) holds for $\mu$, then there exist $t \in \mathbb{T}$, $r, b \in \mathbb{Z}, l \in \mathbb{N}^{+}, s_{1}, \ldots, s_{l} \in \mathbb{Z}^{*}$, and a function $\zeta: \mathbb{T} \rightarrow \mathbb{\mathbb { T }}$ such that $T_{r_{0}} \circ \zeta=$ id and

$$
\sigma_{x}=\delta(t) * T_{r}^{-1}\left[\delta(b \zeta(x)) * T_{s_{1}} \nu * \cdots * T_{s_{i}} \nu\right] \quad \mu \text {-a.e. }
$$

This follows from the following general observations.

First, if $\mu=\delta\left(t^{\prime}\right) * \nu, e\left(-r m_{j} x\right) \rightarrow \hat{\sigma}_{x, \nu}(r)$ weak $^{*}$ in $L^{\infty}(\nu)$, and $e\left(-r m_{j} x\right) \rightarrow \hat{\sigma}_{x, \mu}(r)$ weak $^{*}$ in $L^{\infty}(\mu)$, then let $\left\{m_{j}^{\prime}\right\} \subset\left\{m_{j}\right\}$ be such that $m_{j}^{\prime} t^{\prime} \rightarrow t$. It is easy to see that

$$
\sigma_{x, \mu}=\delta(t) * \sigma_{x-t^{\prime}, \nu} \quad \mu \text {-a.e. }
$$

Second, if $\mu=T_{r_{0}} \nu\left(r_{0} \neq 0\right)$, then using the same notation, we claim that

$$
\sigma_{x, \mu}=T_{r_{0}} \sigma_{\zeta(x), \nu} \quad \mu \text {-a.e. }
$$

for some function $\zeta: \mathbb{T} \rightarrow \mathbb{T}$ with $T_{r_{0}} \circ \zeta=$ id. For we have $\left\{m_{j} x\right\} \sim \sigma_{x, \nu} \nu$-a.e. without loss of generality; let $E=\left\{x:\left\{m_{j} x\right\} \sim \sigma_{x, \nu}\right\}$. Since $\nu E=1$, we have $\mu T_{r_{0}} E=1$. Let $\zeta: \mathbb{T} \rightarrow \mathbb{T}$ be any map such that $\zeta(x) \in E$ for $x \in T_{r_{0}} E$ and $T_{r_{0}} \circ \zeta=$ id. Then for $\mu$-a.e. $x$, we have $\zeta(x) \in E$, so that $\left\{m_{j} \zeta(x)\right\} \sim \sigma_{\zeta(x), \nu}$, whence $\left\{m_{j} x\right\}=\left\{r_{0} m_{j} \zeta(x)\right\} \sim T_{r_{0}} \sigma_{\zeta(x), \nu}$, as desired. 
Now if $\nu$ is $q$-invariant and satisfies (5) and (6), suppose that $\mu=\delta\left(t^{\prime}\right) * T_{r_{0}} \nu$, $r_{0} \neq 0$. With notation as above, we have

$$
\sigma_{x, \mathcal{A}}=\delta\left(t_{1}\right) * \sigma_{x-t^{\prime}, T_{r_{0} \nu}}=\delta\left(t_{1}\right) * T_{r_{0}} \sigma_{\zeta_{1}\left(x-t^{\prime}\right), \nu} \quad \mu \text {-a.e. }
$$

for some $t_{1} \in \mathbb{T}$ and some $\zeta_{1}$ with $T_{r_{0}} \circ \zeta_{1}=$ id. By (7), we may then write

$$
\begin{aligned}
\sigma_{x, \mu} & =\delta\left(t_{1}\right) * T_{r_{0}}\left[T_{r^{\prime}}^{-1}\left[\delta\left(b^{\prime} \zeta_{1}\left(x-t^{\prime}\right)\right) * T_{s_{1}^{\prime}} \nu * \cdots * T_{s i} \nu\right]\right] \quad \mu \text {-a.e. } \\
& =\delta\left(t_{1}\right) * T_{p} T_{r}^{-1}\left[\delta\left(b^{\prime} \zeta_{1}\left(x-t^{\prime}\right)\right) * T_{s_{1}^{\prime}} \nu * \cdots * T_{s_{i}^{\prime}} \nu\right] \mu \text {-a.e., }
\end{aligned}
$$

where $r$ and $p$ are relatively prime. In this case, $T_{p}$ and $T_{r}^{-1}$ commute, so that

$$
\sigma_{x, \mu}=\delta\left(t_{1}\right) * T_{r}^{-1}\left[\delta\left(p b^{\prime}\left(\zeta(x)+t_{2}\right)\right) * T_{p s_{1}^{\prime}} \nu * \cdots * T_{p s i} \nu\right] \quad \mu \text {-a.e., }
$$

where $t_{2}$ is chosen as any (fixed) point such that $T_{r_{0}} t_{2}=-t^{\prime}$ and $\zeta(x)=\zeta_{1}\left(x-t^{\prime}\right)-t_{2}$; we then have $T_{r_{0}} \circ \zeta=\mathrm{id}$. Therefore

$$
\sigma_{x, \mu}=\delta(t) * T_{r}^{-1}\left[\delta(b \zeta(x)) * T_{s_{1}} \nu * \cdots * T_{s_{l}} \nu\right] \quad \mu \text {-a.e., }
$$

where $t$ is any point such that $T_{r}\left(t-t_{1}\right)=p b^{\prime} t_{2}, b=p b^{\prime}$ and $s_{i}=p s_{i}^{\prime}(1 \leq i \leq l)$. This gives (13).

Other examples of $q$-invariant measures satisfying (5) and (6) are given by generalized Riesz products: if $P(x)$ is a trigonometric polynomial

$$
P(x)=1+\operatorname{Re}\left\{\sum_{n=D_{1}}^{D_{2}} \alpha_{n} e(n x)\right\}
$$

satisfying $P(x) \geq 0,0<D_{2} / D_{1} \leq(|q|-1) / 2$, and $\alpha_{n}=0$ if $q \mid n$, then $\mu=\prod_{k \geq 0} P\left(q^{k} x\right)$ is seen to be $q$-invariant and to satisfy (5) and (6). In general, if $\nu$ is a $q$-invariant measure satisfying (5) and (6), then so is $\mu=T_{r_{0}}^{-1} \nu$ for any $r_{0}$ relatively prime to $q$.

We wonder whether hypothesis (6) can be eliminated from Theorem 1, subject to an appropriate modification of (7).

\section{Products of transformations}

In the non-invariant case, the following kind of phenomenon occurs. Suppose that

$$
\mu=\prod_{k \geq 0}\left(1+\operatorname{Re}\left\{\alpha_{k} e\left(q^{k} x\right)\right\}\right)
$$

and $\alpha_{k} \rightarrow \alpha$; then $\left\{q^{k} x\right\} \sim \rho \mu$-a.e., where $\rho=\prod_{k \geq 0}\left(1+\operatorname{Re}\left\{\alpha e\left(q^{k} x\right)\right\}\right)$. Although $\rho$ may be singular to $\mu$, nevertheless $\rho$ is clearly closely related to $\mu$. Once we give up invariance, our problem is almost as easy to treat for products of transformations $T_{q_{n}} T_{q_{n-1}} \cdots T_{q_{1}}$ as for iterates $T_{q}^{n}$. Thus, we proceed directly to his general case.

Given $\left|q_{n}\right| \geq 2, Q_{n}=q_{1} q_{2} \cdots q_{n}, Q_{0}=1$, let $\alpha(m)$ be the largest integer $\alpha$ such that $Q_{\alpha} \mid m$; put $\alpha(0)=0$. We denote

thus $\delta_{n}(m) \neq 0$ if $m n \neq 0$.

$$
\delta_{n}(m)=\left|\frac{m}{Q_{\alpha(m)}}-\frac{Q_{n+\alpha(m)}}{Q_{\alpha(m)}} \mathbb{Z}\right|
$$

THEOREM 3. Let $\mu$ be a probability measure, $\left|q_{n}\right| \geq 2$, $\sup _{n}\left|q_{n}\right|<\infty, Q_{n}=q_{1} q_{2} \cdots q_{n}$, $Q_{0}=1$. Suppose that

$$
\forall b \in \mathbb{Z} \lim _{n \rightarrow \infty} \sup _{\substack{a \in \mathbb{Z} \\ p \in \mathbb{N}}}\left|\hat{\mu}\left(a Q_{n+p}+b Q_{p}\right)-\hat{\mu}\left(a Q_{n+p}\right) \hat{\mu}\left(b Q_{p}\right)\right|=0
$$




$$
\text { if } \lim _{n \rightarrow \infty} \lim _{j \rightarrow \infty} \delta_{n}\left(m_{j}\right)=\infty \text {, then } e\left(m_{j} x\right) \stackrel{w^{*}}{\rightarrow} 0 \quad \text { in } L^{\infty}(\mu)
$$

and

$$
\varlimsup_{n \rightarrow \infty}|\hat{\mu}(n)|<1 .
$$

Then if $\left|m_{j}\right| \rightarrow \infty$ is such that (3) holds, there exist $r, b \in \mathbb{Z}, l \in \mathbb{N}^{+}$, and $s_{1}, \ldots, s_{l} \in \mathbb{Z}^{*}$ such that

$$
\sigma_{x}=T_{r}^{-1}\left[\delta(b x) *\left(\underset{i=1}{*} T_{s_{i}} \nu_{i}\right)\right] \mu \text {-a.e., }
$$

where for each $i$, there is a sequence $n_{j} \uparrow \infty$ such that

$$
\forall k \in \mathbb{Z} \quad \hat{\mu}\left(k Q_{n_{i}}\right) \rightarrow \hat{\nu}_{i}(k) .
$$

In other words, $\sigma_{x}$ is obtained from the weak ${ }^{*}$ limit points of $\left\{T_{Q_{n}} \mu\right\}_{n \geq 0}$.

We shall first establish

LEMMA 4. Let $\sup \left|q_{n}\right|<\infty$ and $\left\{m_{j}\right\}$ be a sequence of integers such that for all $n \geq 1$ and all $r, \lim _{j \rightarrow \infty} \delta_{n}\left(r m_{j}\right)$ exists. Then

$$
E=\left\{r: \lim _{n \rightarrow \infty} \lim _{j \rightarrow \infty} \delta_{n}\left(r m_{j}\right)<\infty\right\}
$$

is a subgroup of $\mathbb{Z}$.

Proof. If $E=\{0\}$, there is nothing to prove. Otherwise, let $r, s \in E, r \neq s$. Since $\lim _{j \rightarrow \infty} \delta_{n}\left(r m_{j}\right)$ is constant for large $n$, we may write

$$
\forall n \forall^{e} j \quad r m_{j}=a_{j, n} Q_{n+\alpha\left(r m_{j}\right)}+b Q_{\alpha\left(r m_{j}\right)},
$$

where ' $\forall^{e} j$ ' means 'for all but a finite number of $j$ ' (cf. [6]). Likewise, we may write

$$
\forall n \forall^{e} j \quad s m_{j}=a_{j, n}^{\prime} Q_{n+\alpha\left(s m_{j}\right)}+b^{\prime} Q_{\alpha\left(s m_{j}\right)} .
$$

Furthermore, by taking a subsequence of $\left\{m_{j}\right\}$ if necessary, we may assume that either $\forall j \alpha\left(r m_{j}\right)=\alpha\left(s m_{j}\right)$ or $\forall j \alpha\left(r m_{j}\right)>\alpha\left(s m_{j}\right)$, and that either $\alpha\left(r m_{j}\right)-\alpha\left(s m_{j}\right) \rightarrow \infty$ or $\left\{\alpha\left(r m_{j}\right)-\alpha\left(s m_{j}\right)\right\}$ is bounded.

Suppose first that $\alpha\left(r m_{j}\right)>\alpha\left(s m_{j}\right)$. Then $\alpha\left((r-s) m_{j}\right)=\alpha\left(s m_{j}\right)$ and

$$
\forall n \forall^{e} j \quad(r-s) m_{j}=a_{j, n}^{\prime \prime} Q_{n+\alpha\left(s m_{j}\right)}+b^{\prime \prime} Q_{\alpha\left(s m_{j}\right)},
$$

where $b^{\prime \prime}=-b^{\prime}$ if $\alpha\left(r m_{j}\right)-\alpha\left(s m_{j}\right) \rightarrow \infty$ and $\forall^{e} j b^{\prime \prime}=b Q_{\alpha\left(r m_{j}\right)} Q_{\alpha\left(s m_{j}\right)}^{-1}-b^{\prime}$ if $\alpha\left(r m_{j}\right)-$ $\alpha\left(s m_{j}\right)$ is bounded. Hence $r-s \in E$.

Now suppose that $\alpha\left(r m_{j}\right)=\alpha\left(s m_{j}\right)$. We claim that $b \neq b^{\prime}$. For if $b=b^{\prime}$, then multiplying (20) by $s,(21)$ by $r$, and subtracting, we obtain that

$$
\left.\forall n \forall^{e} j \quad Q_{n+\alpha\left(s m_{j}\right)}\right)(r-s) b Q_{\alpha\left(s m_{j}\right)} .
$$

This contradicts the fact that $r \neq s$ and $b \neq 0$. Since

$$
\forall n \forall^{e} j \quad(r-s) m_{j}=\left(a_{j, n}-a_{j, n}^{\prime}\right) Q_{n+\alpha\left(s m_{j}\right)}+\left(b-b^{\prime}\right) Q_{\alpha\left(s m_{j}\right)},
$$

it follows that $r-s \in E$.

The proof now proceeds essentially as for Theorem 1 and we restrict ourselves to its outline. 
Proof of Theorem 3. We take $\sigma_{x} \neq \lambda$ and assume that $\lim _{j \rightarrow \infty} \delta_{n}\left(r m_{j}\right)$ exists for all $n$ and $r$. Then the set $E$ in (19) is equal to $r_{0} \mathbb{Z}$ for some $r_{0}>0$. By (15), there exists $\nu_{x}$ such that $\sigma_{x}=-T_{\Im_{0}}^{-1} \nu_{x} \mu$-a.e.

We may suppose that $r_{0} m_{j}=a_{j} Q_{n_{j}+\alpha\left(r_{0} m_{j}\right)}+s_{0} Q_{\alpha\left(r_{0} m_{j}\right)}$, that $n_{j} \rightarrow \infty$, that $\left\{e\left(r a_{j} Q_{n_{j}+\alpha\left(r_{0} m_{j}\right)} x\right)\right\}_{j}$ has a weak* limit in $L^{\infty}(\mu)$ for each $r$, and that $\left\{\alpha\left(r_{0} m_{j}\right)\right\}$ is either constant or tends to $\infty$. If $\left\{\alpha\left(r_{0} m_{j}\right)\right\}$ is constant, let

$$
\hat{\Sigma}_{1}(r)=\lim _{j \rightarrow \infty} \hat{\mu}\left(r a_{j} Q_{n_{j}+\alpha\left(r_{0} m_{j}\right)}\right) \quad \text { and } \quad b_{0}=s_{0} Q_{\alpha\left(r_{0} m_{j}\right)}
$$

otherwise, let $\hat{\Sigma}_{1}(r)=\lim _{j \rightarrow \infty} \hat{\mu}\left(r r_{0} m_{j}\right)$ and $b_{0}=0$. Then $\nu_{x}=\delta\left(b_{0} x\right) * \Sigma_{1} \mu$-a.e. by (14).

Define $m_{j}^{(1)}=r_{0} m_{j}-b_{0}$; thus, $\hat{\Sigma}_{1}(r)=\lim _{j \rightarrow \infty} \hat{\mu}\left(r m_{j}^{(1)}\right)$. We can argue as in the first paragraph to write $\Sigma_{1}=T_{r_{1}}^{-1} \Sigma_{1}^{\prime}$, where, without loss of generality, we may assume that

$$
r_{1} m_{j}^{(1)}=a_{j}^{(2)} Q_{n_{j}+\alpha\left(r_{1} m_{j}^{(1)}\right)}+s_{1}^{\prime} Q_{\alpha\left(r_{1} m_{j}^{(1)}\right)}
$$

and that $\Sigma_{1}^{\prime}=T_{s_{i}} \nu_{1} * \Sigma_{2}$, where $\hat{\nu}_{1}(r)=\lim _{j \rightarrow \infty} \hat{\mu}\left(r Q_{\alpha\left(r_{1} m_{j}^{(1)}\right)}\right)$. Note that $s_{1}^{\prime} \neq 0$. We then define

$$
m_{j}^{(2)}=r_{1} m_{j}^{(1)}-s_{1}^{\prime} Q_{\alpha\left(r_{1} m_{j}^{(1)}\right)}
$$

and proceed for $\Sigma_{2}$ as we did for $\Sigma_{1}$, etc. This process ends since $\sigma_{x} \neq \lambda$ and (16) holds.

We thus obtain the expression

$$
\sigma_{x}=T_{r_{0}}^{-1}\left[\delta\left(b_{0} x\right) * T_{r_{1}}^{-1}\left[T_{s_{1}^{\prime}}^{-1} \nu_{1} * T_{r_{2}}^{-1}\left[T_{s_{2}} \nu_{2} * \cdots * T_{r_{l}}^{-1} T_{s_{i}} \nu_{l}\right]\right] \cdots\right] \mu \text {-a.e., }
$$

which is reduced to (17) by use of (4).

Remark. Even if (16) does not hold, we may still conclude that

$$
\sigma_{x}=T_{r}^{-1}\left[\delta(b x) * T_{s} \nu * \Sigma\right] \quad \mu \text {-a.e., }
$$

where $\nu$ has the form $(18)$ and $\hat{\Sigma}(k)=\lim _{j} \hat{\mu}\left(k l_{j}\right)$ for some sequence $\left\{l_{j}\right\}$ (not necessarily tending to $\infty$ ).

The most obvious example of a measure satisfying the hypotheses of Theorem 3 is a Riesz product

$$
\mu=\prod_{k \geq 0}\left(1+\operatorname{Re}\left\{\alpha_{k} e\left(Q_{k} x\right)\right\}\right)
$$

with $\left|\alpha_{k}\right| \leq 1$ arbitrary, $Q_{k}\left|Q_{k+1},\right| Q_{k+1} / Q_{k} \mid \geq 3$, and $\sup _{k}\left|Q_{k+1} / Q_{k}\right|<\infty$. In this case, the measures $\nu_{i}$ of (17) are also Riesz products $\prod_{k \geq 0}\left(1+\operatorname{Re}\left\{\beta_{k} e\left(P_{k} x\right)\right\}\right)$, with each $\beta_{k}$ a limit point of $\left\{\alpha_{j}\right\}, P_{0}=1, P_{k} \mid P_{k+1}$, and each $P_{k+1} / P_{k}$ a limit point of $\left\{Q_{j+1} / Q_{j}\right\}$.

Consider next the measure

$$
\mu=\underset{k \geq 1}{*}\left[p_{0, k} \delta(0)+p_{1, k} \delta\left(Q_{k}^{-1}\right)+\cdots+p_{\left|q_{k}\right|-1, k} \delta\left(\left(\left|q_{k}\right|-1\right) Q_{k}^{-1}\right)\right],
$$

where

$$
\left|q_{k}\right| \geq 2, \quad \sup \left|q_{k}\right|<\infty, \quad Q_{k}=q_{1} q_{2} \cdots q_{k}, \quad p_{i, k} \geq 0, \quad \text { and } \quad \sum_{i=1}^{\left|q_{k}\right|-1} p_{i, k}=1 .
$$

We claim that $\mu$ satisfies (14), (15) and (16) if $\operatorname{gcd}\left\{i-i_{0}: i \in I\right\}=1$ for some set $I$ and some $i_{0} \in I$, where $I$ satisfies the property that $\exists \varepsilon>0 \exists K \forall k_{0} \exists k \in\left[k_{0}, k_{0}+K[\right.$ $\forall i \in I p_{i, k} \geq \varepsilon$; here, we interpret $p_{i, k}=0$ if $i \geq\left|q_{k}\right|$. (This is the case in particular for

$$
\mu=\underset{k \geq 1}{*}\left[p_{k} \delta(0)+\left(1-p_{k}\right) \delta\left(2^{-k}\right)\right]
$$

if $\exists \varepsilon>0 \exists K \forall k_{0} \exists k \in\left[k_{0}, k_{0}+K\left[\min \left\{p_{k},\left(1-p_{k}\right)\right\} \geq \varepsilon\right.\right.$. 
This example will be important later.) Now since $\mu$ is continuous, (14) is proved just as (5) is proved for ordinary invariant Bernoulli convolutions (12) (that is, by 'lifting' to a product measure). To prove (15), suppose that $\hat{\mu}\left(m_{j}\right)$ has a non-zero limit. By taking an appropriate subsequence of $\left\{m_{j}\right\}$, we may assume that all the limits encountered below exist. For certain $\theta_{k, j}$, we have

$$
\begin{aligned}
\infty & >\lim _{j \rightarrow \infty} \sum_{k \geq 1}\left[1-\left|\sum_{i=0}^{\left|a_{k}\right|-1} p_{i, k} e\left(-i m_{j} Q_{k}^{-1}\right)\right|\right] \\
& =\lim _{j} \sum_{k \geq 1}\left[1-\sum_{i} p_{i, k} e\left(-i m_{j} Q_{k}^{-1}\right) e\left(\theta_{k, j}\right)\right] \\
& =\lim _{j} \sum_{k \geq 1} \sum_{i} p_{i, k}\left[1-e\left(-i m_{j} Q_{k}^{-1}\right) e\left(\theta_{k, j}\right)\right] \\
& \geq \lim _{j} \sum_{k \geq 1} \sum_{i} p_{i, k+\alpha\left(m_{j}\right)} \operatorname{Re}\left\{1-e\left(-i m_{j} Q_{k+\alpha\left(m_{j}\right)}^{-1}\right) e\left(\theta_{k+\alpha\left(m_{j}\right), j}\right)\right\} .
\end{aligned}
$$

Now for $j \geq 1$ and $l \geq 0, \exists k=k(l, j) \in\left[l K+\alpha\left(m_{j}\right),(l+1) K+\alpha\left(m_{j}\right)[\right.$ such that $\forall i \in I$ $p_{i, k(l, j)} \geq \varepsilon$. Hence for $i \in I$,

$$
\begin{aligned}
\infty & >\lim _{j} \sum_{l \geq 0} \varepsilon \operatorname{Re}\left\{1-e\left(-i m_{j} Q_{k(l, j)}^{-1}\right) e\left(\theta_{k(l, j), j}\right)\right\} \\
& \geq \varepsilon \sum_{l \geq 0} \lim _{j} \operatorname{Re}\left\{1-e\left(-i m_{j} Q_{k(l, j)}^{-1}\right) e\left(\theta_{k(l, j), j}\right)\right\} .
\end{aligned}
$$

Therefore for $i \in I$,

whence

$$
\lim _{i \rightarrow \infty} \lim _{j \rightarrow \infty} \operatorname{Re}\left\{1-e\left(-i m_{j} Q_{k(l, j)}^{-1}\right) e\left(\theta_{k(l, j), j}\right)\right\}=0,
$$

$$
\lim _{l} \lim _{j} e\left(-i m_{j} Q_{k(l, j)}^{-1}\right) e\left(\theta_{k(l, j), j}\right)=1 .
$$

The hypothesis $\operatorname{gcd}\left(I-i_{0}\right)=1$ implies finally that

$$
\lim _{i} \lim _{j} e\left(m_{j} Q_{k(l, j)}^{-1}\right)=1 \text {. }
$$

Now if (15) were not true, in other words, if $\lim _{k} \lim _{j} \delta_{k}\left(m_{j}\right)=\infty$, then for all $N$ there would be a $k_{0}=k_{0}(N)$ such that $N_{1}=\lim _{j} \delta_{k_{0}}\left(m_{j}\right) \geq N$. Let $\delta_{k_{0}}\left(m_{j}\right)=N_{1}$ for $j \geq j_{0}=j_{0}(N)$ and, for $j \geq j_{0}$, let $n_{j}=n_{j}(N)$ be the least integer such that $\left|Q_{n_{j}+\alpha\left(m_{j}\right)} Q_{\alpha\left(m_{j}\right)}^{-1}\right| / 2 \geq N_{1}$. Since $n_{j}<2+\log _{2} N_{1}$, we may choose an infinite sequence $\mathscr{J}=\mathscr{J}(N) \subset\left[j_{0}, \infty\left[\right.\right.$ such that $n_{j}$ is equal to a fixed $n$ for $j \in \mathscr{J}$. Let $l=l(N)$ be the least integer such that $I K \geq n$ and consider any $j \in \mathscr{J}$. We have

$$
\left\|m_{j} Q_{k(l, j)}^{-1}\right\|=\left|Q_{k(l, j)}^{-1} Q_{\alpha\left(m_{j}\right)}\right| \delta_{k(l, j)-\alpha\left(m_{j}\right)}\left(m_{j}\right)
$$

Now $k_{0} \geq n$; for $k_{0} \geq k \geq n$, we have $\delta_{k}\left(m_{j}\right)=N_{1}$, while for $k>k_{0}$, we have $\delta_{k}\left(m_{j}\right) \geq$ $N_{1}$. Since $k(l, j)-\alpha\left(m_{j}\right) \geq l K \geq n$, it follows that

$$
\left\|m_{j} Q_{k(l, j)}^{-1}\right\| \geq\left|Q_{n+\alpha\left(m_{j}\right)}^{-1} Q_{\alpha\left(m_{j}\right)} N_{1}\right|\left|Q_{n+\alpha\left(m_{j}\right)} Q_{k(l, j)}^{-1}\right| .
$$

By choice of $n$, the first term on the right is greater than $1 /(2 q)$, where $q=\sup _{k}\left|q_{k}\right|$. In addition, since

$$
k(l, j)-n-\alpha\left(m_{j}\right)<2 K+(l-1) K-n<2 K,
$$

the second term on the right is at least $q^{-2 K+2}$. Therefore $\left\|m_{j} Q_{k(l, j)}^{-1}\right\|>q^{-2 K+1} / 2$. Since this is true for $j \in \mathscr{J}$ and since $l(N) \rightarrow \infty$ as $N \rightarrow \infty$, it follows that

$$
\lim _{l} \lim _{j}\left\|m_{j} Q_{k(l, j)}^{-1}\right\| \geq \frac{1}{2} q^{-2 K+1},
$$


which contradicts (22). This proves (15). Finally, to establish (16), we will show that for $n \neq 0$,

$$
|\hat{\mu}(n)| \leq 1-4 \varepsilon q^{-2 K} \text {. }
$$

Given $n \neq 0$, let $k=\alpha(n)+1$. Then $n Q_{k}^{-1}=N+r q_{k}^{-1}$ for some integers $N$ and $r$, $0<r<\left|q_{k}\right|$. Since $\operatorname{gcd}\left(I-i_{0}\right)=1$, there is some $i_{1} \in I$ such that $\left(i_{1}-i_{0}\right) n Q_{k}^{-1} \notin \mathbb{Z}$, whence $\left\|\left(i_{1}-i_{0}\right) n Q_{k}^{-1}\right\| \geq\left|q_{k}\right|^{-1}$. Furthermore, for some $l \in\left[k, k+K\left[, p_{i_{0}, l} \geq \varepsilon\right.\right.$ and $p_{i_{1}, l} \geq \varepsilon$. We have

$$
\begin{aligned}
|\hat{\mu}(n)| & \leq\left|\sum_{i=0}^{\left|q_{l}\right|-1} p_{i, l} e\left(-i n Q_{l}^{-1}\right)\right| \\
& =\left|\sum_{i=0}^{\left|q_{l}\right|-1} p_{i, l} e\left(-\left(i-i_{0}\right) n Q_{l}^{-1}\right)\right| \\
& \leq\left|p_{i_{0}, l}+p_{i_{1}, l} e\left(-\left(i_{1}-i_{0}\right) n Q_{l}^{-1}\right)\right|+1-p_{i_{0}, l}-p_{i_{1}, l}
\end{aligned}
$$

Now for real $x, y$ and $\theta$, we have

$$
\begin{aligned}
|x+y e(\theta)| & =\left[(x+y)^{2}-4 x y \sin ^{2} \pi \theta\right]^{1 / 2} \leq\left[(x+y)^{2}-16 x y\|\theta\|^{2}\right]^{1 / 2} \\
& \leq(x+y)-8 \frac{x y}{x+y}\|\theta\|^{2} .
\end{aligned}
$$

Therefore

$$
|\hat{\mu}(n)| \leq 1-8 \frac{p_{i_{0}, l} p_{i_{1}, l}}{p_{i_{0}, l}+p_{i_{1}, l}}\left\|\left(i_{1}-i_{0}\right) n Q_{l}^{-1}\right\|^{2}
$$

Our choice of $l$ ensures (23).

Theorem 3 admits a ready, if somewhat ungainly, extension to the case of unbounded $q_{n}$.

THEOREM 5. Let $\mu$ be a probability measure, $\left|q_{n}\right| \geq 2, Q_{n}=q_{1} q_{2} \cdots q_{n}$, and $Q_{0}=1$. Suppose that

$$
\begin{gathered}
\forall U \in \mathbb{N} \quad \forall b_{0}, \ldots, b_{U} \in \mathbb{Z} \\
\lim _{n \rightarrow \infty} \sup _{\substack{a \in \mathbb{Z} \\
p \in \mathbb{N}}}\left|\hat{\mu}\left(a Q_{n+p}+\sum_{u=0}^{U} b_{u} Q_{u+p}\right)-\hat{\mu}\left(a Q_{n+p}\right) \hat{\mu}\left(\sum_{u=0}^{U} b_{u} Q_{u+p}\right)\right|=0, \\
\text { if } e\left(m_{j} x\right) \nrightarrow 0 \text { weak }^{*} \text { in } L^{\infty}(\mu), \text { then there exist }\left\{m_{j}^{\prime}\right\} \subset\left\{m_{j}\right\}, U, \\
n_{j} \in \mathbb{N}, a_{j}, b_{0}, \ldots, b_{U} \in \mathbb{Z} \text { such that } n_{j} \rightarrow \infty \text { and } \\
m_{j}^{\prime}=a_{j} Q_{n_{j}+\alpha\left(m_{j}^{\prime}\right)}+\sum_{u=0}^{U} b_{u} Q_{u+\alpha\left(m_{j}^{\prime}\right)},
\end{gathered}
$$

and

$$
\varlimsup_{n \rightarrow \infty}|\hat{\mu}(n)|<1 .
$$

If $\left|m_{j}\right| \rightarrow \infty$ is such that (3) holds, then there exist $r, b \in \mathbb{Z}$ and $l \in \mathbb{N}^{+}$such that

$$
\sigma_{x}=T_{r}^{-1}\left[\delta(b x) *\left(\begin{array}{cc}
1 & \\
i=1 & \nu_{i}
\end{array}\right)\right] \mu \text {-a.e., }
$$


where for each $i$, there is a sequence $n_{j} \uparrow \infty, U \in \mathbb{N}$, and $b_{0}, \ldots, b_{U} \in \mathbb{Z}$ such that

$$
\forall k \in \mathbb{Z} \quad \hat{\mu}\left(k \sum_{u=0}^{U} b_{u} Q_{u+n_{j}}\right) \rightarrow \hat{\nu}_{i}(k) .
$$

The proof is exactly parallel; we shall only remark that the analogue of Lemma 4 is the following:

LEMMA 6. Let $\left\{m_{j}\right\}$ be a sequence of integers such that for all $r \in \mathbb{Z}$, either $\left\{r m_{j}\right\}$ is of the form

$$
r m_{j}=a_{j} Q_{n_{j}+\alpha\left(r m_{j}\right)}+\sum_{u=0}^{U} b_{u} Q_{u+\alpha\left(r m_{j}\right)} \quad\left(n_{j} \rightarrow \infty\right),
$$

or $\left\{r m_{j}\right\}$ has no subsequence of this form. Then the set $E$ of $r \in \mathbb{Z}$ with $\left\{r m_{j}\right\}$ of the above form is a subgroup of $\mathbb{Z}$.

As an application, we consider any Riesz product

$$
\mu=\prod_{k \geq 0}\left(1+\operatorname{Re}\left\{\alpha_{k} e\left(Q_{k} x\right)\right\}\right)
$$

with $Q_{k} \mid Q_{k+1}$ and $\left|Q_{k+1} / Q_{k}\right| \geq 3$. The hypotheses (24)-(26) are evidently satisfied and it remains to identify the measures $\nu_{i}$ of (28). Fix integers $U, b_{0}, \ldots, b_{U}$, and $n_{j} \uparrow \infty$, let $B_{j}=\sum_{u=0}^{U} b_{u} Q_{u+n_{j}}$, and assume that $\nu$ is the weak* limit of $T_{B_{j}} \mu$. Clearly, we may assume that $\sum_{u=0}^{U^{\prime}} b_{u} Q_{u+n_{i}} \neq 0$ for $U^{\prime} \leq U$ and all $j$. Furthermore, we may assume that for all $k \geq 0,\left\{Q_{k+n_{j}} Q_{n_{j}}^{-1}\right\}_{j}$ has a finite or infinite limit and that $\left\{\alpha_{k+n_{j}}\right\}_{j}$ has a limit. There are two possibilities: either for all $k \geq 1, \lim _{j \rightarrow \infty} Q_{k+n_{j}} Q_{n_{j}}^{-1}$ is finite or not. The former case is easily handled: $\nu=T_{s} \nu^{\prime}$, where

$$
\begin{gathered}
s=\lim _{j \rightarrow \infty} B_{j} Q_{n_{j}}^{-1}, \nu^{\prime}=\prod_{k \geq 0}\left(1+\operatorname{Re}\left\{\tilde{\alpha}_{k} e\left(P_{k} x\right)\right\}\right), \\
P_{k}=\lim _{j \rightarrow \infty} Q_{k+n_{j}} Q_{n_{j}}^{-1}, \quad \text { and } \quad \tilde{\alpha}_{k}=\lim _{j \rightarrow \infty} \alpha_{k+n_{j}} .
\end{gathered}
$$

On the other hand, we claim that in the latter case, the spectrum of $\nu$ is finite (which is enough for our purposes in $\S 5$ ). Let $k_{0}$ be the smallest integer such that $\lim _{j \rightarrow \infty} Q_{k_{0}+n_{j}} Q_{n_{j}}^{-1}$ is infinite. Set $n_{0}=\lim _{j \rightarrow \infty}\left|Q_{k_{0}-1+n_{j}} Q_{n_{j}}^{-1}\right|$ and define $B_{j}^{\prime}=$ $\sum_{u=0}^{\min \left(k_{0}-1, U\right)} b_{u} Q_{u+n_{j}}, B_{j}^{\prime \prime}=B_{j}-B_{j}^{\prime}$. If $\hat{\nu}(n) \neq 0$, then for sufficiently large $j, \hat{\mu}\left(n B_{j}\right) \neq 0$, which means that $n B_{j}$ has the representation

$$
n B_{j}=\sum_{u \geq 0} \varepsilon_{u}^{(j)} Q_{u+n_{j}}, \quad \varepsilon_{u}^{(j)}=0, \pm 1
$$

Since $Q_{k_{0}+n_{j}}$ divides $n B_{j}^{\prime \prime}$ and $\sum_{u \geq k_{0}} \varepsilon_{u}^{(j)} Q_{u+n_{j}}$, it follows that for sufficiently large $j$, $n B_{j}^{\prime}=\sum_{u=0}^{k_{0}^{-1}} \varepsilon_{u}^{(j)} Q_{u+n_{j}}$. Hence

$$
\left|n Q_{n_{j}}\right| \leq\left|n B_{j}^{\prime}\right| \leq \sum_{u=0}^{k_{0}-1}\left|Q_{u+n_{j}}\right|<(3 / 2)\left|Q_{k_{0}-1+n_{j}}\right| \text {, whence }|n|<(3 / 2) n_{0} .
$$

That is, the spectrum of $\nu$ is contained in the finite set $]-(3 / 2) n_{0},(3 / 2) n_{0}[$, as desired.

\section{Perturbed Riesz products}

The above ideas can be used to analyze the asymptotic distribution of sequences relative to Riesz products based on a set of perturbed frequencies. The simplest 
example is

with $\alpha_{k} \rightarrow \alpha$. Set

$$
\mu=\prod_{k \geq 0}\left(1+\operatorname{Re}\left\{\alpha_{k} e\left[\left(q^{k}+d\right) x\right]\right\},\right.
$$

$$
\rho_{t}=\prod_{k \geq 0}\left(1+\operatorname{Re}\left\{\alpha e(d t) e\left(q^{k} x\right)\right\}\right)
$$

for $t \in \mathbb{T}$. Then if (3) holds, we can write

$$
\sigma_{t}=T_{r}^{-1}\left[\delta(b t) * T_{s_{1}} \rho_{t} * \cdots * T_{s_{t}} \rho_{t}\right] \quad \mu \text {-a.e. }
$$

In fact, we shall treat the following more general case. Let

$$
\mu=\prod_{k \geq 0}\left(1+\operatorname{Re}\left\{\alpha_{k} e\left[\left(Q_{k}+d_{k}\right) x\right]\right\}\right),
$$

where $\left|\alpha_{k}\right| \leq 1, Q_{k}\left|Q_{k+1}, \quad\right| Q_{k+1} / Q_{k} \mid \geq 3$, and $\left|d_{k}\right|$ is bounded. Set $\mu^{\prime}=$ $\prod_{k \geq 0}\left(1+\operatorname{Re}\left\{\alpha_{k} e\left(Q_{k} x\right)\right\}\right)$. The fundamental observation is that $e\left(m_{j} t\right) \nrightarrow 0$ weak* in $L^{\infty}(\mu)$ if and only if $e\left(m_{j} t\right) \nrightarrow 0$ weak ${ }^{*}$ in $L^{\infty}\left(\mu^{\prime}\right)$. Indeed, $e\left(m_{j} t\right) \nrightarrow 0$ weak ${ }^{*}$ in $L^{\infty}(\mu)$ iff there are a subsequence $\left\{m_{j}^{\prime}\right\} \subset\left\{m_{j}\right\}$, a sequence $\left\{u_{j}\right\}$ tending to $\infty$ and $\varepsilon_{u, j}=0, \pm 1$ such that

$$
m_{j}^{\prime}=\sum_{u \supseteq u_{j}} \varepsilon_{u, j}\left(Q_{u}+d_{u}\right)+\mathrm{O}(1)
$$

and

$$
\liminf _{j \rightarrow \infty}\left|\prod_{u \geq u_{j}}\left(\frac{1}{2} \alpha_{u}\right)^{\left(\varepsilon_{u, j}\right)}\right|>0,
$$

where we denote, for complex $z$,

$$
z^{(\varepsilon)}= \begin{cases}z & \text { if } \varepsilon=1, \\ \bar{z} & \text { if } \varepsilon=-1 \\ 1 & \text { if } \varepsilon=0\end{cases}
$$

But by (31), $\sum_{u \geq u_{j}}\left|\varepsilon_{u, j}\right|=\mathrm{O}(1)$, whence (30) is equivalent (under (31)) to $m_{j}^{\prime}=$ $\sum_{u \geq u_{j}} \varepsilon_{u, j} Q_{u}+\mathrm{O}(1)$; i.e. (30) is then independent of $\left\{d_{k}\right\}$. This establishes our claim.

Our next observation is that an analogue of mixing occurs. Let $U \geq 0, a_{j}$, $b_{0}, \ldots, b_{U} \in \mathbb{Z}, p_{j} \in \mathbb{N}$, and $n_{j} \rightarrow \infty$. Write $B_{j}=\sum_{u=0}^{U} b_{u} Q_{u+p_{j}}$ and assume that the following weak* limits exist in $L^{\infty}(\mu)$ :

Then

$$
\begin{gathered}
h(t)=\lim e\left(-\left(a_{j} Q_{n_{j}+p_{j}}+B_{j}\right) t\right), \\
f(t)=\lim e\left(-a_{j} Q_{n_{j}+p_{j}} t\right), \quad g(t)=\lim e\left(-B_{j} t\right) .
\end{gathered}
$$

$$
h=f \cdot g .
$$

To prove this, we may, by taking a subsequence if necessary, assume that the above weak* limits also exist in $L^{\infty}\left(\mu^{\prime}\right)$; denote them by $\tilde{h}, \tilde{f}$, and $\tilde{g}$ respectively. We may also assume that the limits to appear below exist. It is clear that $\tilde{h}=\tilde{f} \cdot \tilde{\mathrm{g}}$. If $\tilde{h} \neq 0$, then $\tilde{f} \neq 0$ and $\tilde{g} \neq 0$, whence we may write

$$
\begin{gathered}
a_{j} Q_{n_{j}+p_{j}}=\sum_{u \geq n_{j}} \varepsilon_{u, j} Q_{u+p_{j}}, \\
B_{j}=\sum_{u=0}^{U^{\prime}} \varepsilon_{u, j}^{\prime} Q_{u+p_{j}}, \\
\varepsilon_{u, j}, \varepsilon_{u, j}^{\prime} \in\{0, \pm 1\}, \\
\sum_{u \geq n_{j}}\left|\varepsilon_{u, j}\right|=O(1) .
\end{gathered}
$$


Therefore

$$
a_{j} Q_{n_{j}+p_{j}}+B_{j}=\sum_{u \geq n_{j}} \varepsilon_{u, j}\left(Q_{u+p_{j}}+d_{u+p_{j}}\right)+C_{j},
$$

where

$$
C_{j}=\sum_{u=0}^{U^{\prime}} \varepsilon_{u, j}^{\prime}\left(Q_{u+p_{j}}+d_{u+p_{j}}\right)-\sum_{u \geqslant n_{j}} \varepsilon_{u, j} d_{u+p_{j}}-\sum_{u=0}^{U^{\prime}} \varepsilon_{u, j}^{\prime} d_{u+p_{j}} .
$$

Noting that $\tilde{f}$ is a constant and that the last two sums in $C_{j}$ are bounded, we see that $h=\tilde{f} G$, where

$$
G(t)=w^{*}-\lim e\left(-C_{j} t\right) \quad \text { in } L^{\propto}(\mu) .
$$

But evaluation of $G$ gives

$$
G(t)=g(t) e(D t)
$$

where $D=\lim \sum_{u \geq n_{j}} \varepsilon_{u, j} d_{u+p_{j}}$. Therefore $h=\tilde{f} g e(D t)=f g$. On the other hand, if $\tilde{h}=0$, then since $\tilde{f}$ is a constant, either $\tilde{f}$ or $\tilde{g}$ is 0 . Therefore $h=0$ and $f$ or $g$ is 0 , whence (32) again holds.

We are now in a position to imitate our preceding proofs in order to determine $\sigma_{t}$ of (3). If $\sigma_{t} \not \equiv \lambda \mu$-a.e., then there is an $r \neq 0$ such that $\hat{\sigma}_{t}(r) \not \equiv 0 \mu$-a.e., whence $e\left(-r m_{j} t\right) \rightarrow 0$ weak* in $L^{\infty}\left(\mu^{\prime}\right)$. The subgroup $E$ described in Lemma 6 therefore has a least positive element, $r_{0}$; let $\sigma_{t}=T_{r_{0}}^{-1} \nu_{t} \mu$-a.e.

We may suppose that

$$
r_{0} m_{j}=a_{j} Q_{n_{j}+\alpha\left(r_{0} m_{j}\right)}+\sum_{u=0}^{U} b_{u} Q_{u+\alpha\left(r_{0} m_{j}\right)}
$$

$n_{j} \rightarrow \infty$, and $\left\{\alpha\left(r_{0} m_{j}\right)\right\}$ is either constant or tends to $\infty$. If $\alpha\left(r_{0} m_{j}\right)$ is constant, set $b^{\prime}=\sum_{u=0}^{U} b_{u} Q_{u+\alpha\left(r_{0} m_{j}\right)}$; otherwise, set $b^{\prime}=0$. Put $m_{j}^{\prime}=r_{0} m_{j}-b^{\prime}$. Application of (32) to the sequence $\left\{r r_{0} m_{j}\right\}$ shows that

$$
\hat{\nu}_{t}(r)=\hat{\Sigma}_{1, t}(r) e\left(-r b^{\prime} t\right) \quad \mu \text {-a.e., }
$$

where $e\left(-r m_{j}^{\prime} t\right) \rightarrow \hat{\Sigma}_{1, t}(r)$ weak $^{*}$ in $L^{\infty}(\mu)$. Thus $\nu_{t}=\delta\left(b^{\prime} t\right) * \Sigma_{1, t}$.

We may argue as above to write $\Sigma_{1, t}=T_{r_{1}}^{-1} \Sigma_{1, t}^{\prime}$ and, without loss of generality,

$$
r_{1} m_{j}^{\prime}=a_{j}^{\prime} Q_{n_{j}+\alpha\left(r_{1} m_{j}^{\prime}\right)}+\sum_{u=0}^{U^{\prime}} b_{u}^{\prime} Q_{u+\alpha\left(r_{1} m_{j}^{\prime}\right)},
$$

$n_{j}^{\prime} \rightarrow \infty$. We now have $\alpha\left(r_{1} m_{j}^{\prime}\right) \rightarrow \infty$. By (32), $\Sigma_{1, t}^{\prime}=\nu_{1, t}^{\prime} * \Sigma_{2, t}$, where $\hat{\nu}_{1, t}^{\prime}(r)$ is the weak* limit in $L^{\infty}(\mu)$ of $e\left(-r \sum_{u=0}^{U^{\prime}} b_{u}^{\prime} Q_{u+\alpha\left(r_{1} m_{j}\right)} t\right)$ and

$$
\hat{\Sigma}_{2, t}(r)=w^{*}-\lim e\left(-r a_{j}^{\prime} Q_{n_{j}^{\prime}+\alpha\left(r_{1} m_{j}^{\prime}\right)} t\right) .
$$

We proceed for $\Sigma_{2, t}$ as for $\Sigma_{1, t}$ we have $\Sigma_{2, t}=T_{r_{2}}^{-1}\left(\nu_{2, t}^{\prime} * \Sigma_{3, t}\right)$, and so on. Since $\varlimsup|\hat{\lim }| n) \mid \leq \frac{1}{2}<1$, this process ends in a finite number of steps. As before, we conclude that

$$
\sigma_{t}=T_{r}^{-1}\left[\delta(b t) *\left(\begin{array}{cc}
t & \\
i=1 & \nu_{i, t}
\end{array}\right)\right] \mu \text {-a.e., }=
$$

where each $\nu_{i, t}$ is a measure $\omega_{t}$ of the form

$$
\forall k \in \mathbb{Z} \quad e\left(-k \sum_{u=0}^{U} b_{u} Q_{u+n_{j}} t\right) \rightarrow \hat{\omega}_{i}(k) \text { weak* in } L^{\infty}(\mu), \quad n_{j} \rightarrow \infty
$$


We now identify such measures $\omega_{t}$. We base this on the result for $\mu^{\prime}$. We may assume that $\sum_{u=0}^{U^{\prime}} b_{u} Q_{u+n_{j}} \neq 0$ for all $U^{\prime} \leq U$ and all $j$. Let $B_{j}=\sum_{u=0}^{U} b_{u} Q_{u+n_{j}}$ and assume that for al $k \geq 0,\left\{Q_{k+n_{j}} \ddot{Q}_{n_{i}}^{-1}\right\}_{j}$ has a finite or infinite limit, $P_{k}$, and that $\left\{\alpha_{k+n_{j}}\right\}_{j}$ and $\left\{d_{k+n_{j}}\right\}_{j}$ have limits, call then $\tilde{\alpha}_{k}$ and $\tilde{d}_{k}$, respectively. If for some $k \geq 1$, $P_{k}$ is infinite, then we know that $e\left(-k B_{j} t\right) \rightarrow 0$ weak* in $L^{\infty}\left(\mu^{\prime}\right)$ for all but a finite number of $k$; the same is true in $L^{\infty}(\mu)$, so that the spectrum of $\omega_{t}$ is contained in a finite set (independent of $t$ ). On the other hand, if $P_{k}$ is finite for all $k$, then $\omega_{t}=T_{s} \omega_{t}^{\prime}$, where $s=\lim _{j \rightarrow \infty} B_{j} Q_{n_{i}}^{-1}$ and $e\left(-r Q_{n_{j}} t\right) \rightarrow \hat{\omega}_{t}^{\prime}(r)$ weak ${ }^{*}$ in $L^{\infty}(\mu)$. We claim that $\omega_{t}^{\prime}=\rho_{t} \mu$-a.e., where

$$
\rho_{t}=\prod_{k \geq 0}\left(1+\operatorname{Re}\left\{\tilde{\alpha}_{k} e\left(\tilde{d}_{k} t\right) e\left(P_{k} x\right)\right\}\right) .
$$

For if we define

$$
\rho^{\prime}=\prod_{k \geq 0}\left(1+\operatorname{Re}\left\{\tilde{\alpha}_{k} e\left(P_{k} x\right)\right\}\right)
$$

then $e\left(-r Q_{n_{j}} t\right) \rightarrow \hat{\rho}^{\prime}(r)$ weak* in $L^{\infty}\left(\mu^{\prime}\right)$. Thus, if $\hat{\omega}_{t}^{\prime}(r)=0 \mu$-a.e., we have $\hat{\rho}^{\prime}(r)=0$, which implies that $\hat{\rho}_{t}(r)=0$ for all $t$. On the other hand, if $\hat{\omega}_{t}^{\prime}(r) \neq 0$, then $\hat{\rho}^{\prime}(r) \neq 0$, so that we can write $r=\sum_{k \geq 0} \varepsilon_{k} P_{k}, \varepsilon_{k}=0, \pm 1$. Therefore, interpreting limits as weak* in $L^{\infty}(\mu)$, we have

$$
\begin{aligned}
\hat{\omega}_{r}^{\prime}(r) & =\lim _{j \rightarrow \infty} e\left(-\sum_{k \geq 0} \varepsilon_{k} P_{k} Q_{n_{j}} t\right) \\
& =\lim _{j \rightarrow \infty} e\left(-\sum_{k \geq 0} \varepsilon_{k} Q_{k+n_{j}} t\right) \\
& =\left[\lim e\left(-\sum \varepsilon_{k}\left(Q_{k+n_{j}}+d_{k+n_{j}}\right) t\right] e\left(\sum \varepsilon_{k} \tilde{d}_{k} t\right)\right. \\
& =\left[\prod_{k \geq 0} \frac{1}{2} \tilde{\alpha}_{k}^{\left(\varepsilon_{k}\right)}\right] e\left(\sum \varepsilon_{k} \tilde{d}_{k} t\right)=\prod_{k \geq 0}\left[\frac{1}{2} \tilde{\alpha}_{k} e\left(\tilde{d}_{k} t\right)\right]^{\left(\varepsilon_{k}\right)} \\
& =\hat{\rho}_{t}(r) .
\end{aligned}
$$

This shows that $\hat{\omega}_{t}^{\prime}(r)=\hat{\rho}_{t}(r)$ for all $r$, whence the claim.

We sum up our results: either $\sigma_{t}$ is a (non-negative) trigonometric polynomial multiplying $\lambda$,

$$
\sigma_{t}(x)=\left[1+\operatorname{Re} \sum_{n=1}^{N} \beta_{n} e\left(r_{n} t\right) e(n x)\right] \lambda(x),
$$

or $\sigma_{t}$ has the form

$$
\sigma_{t}=T_{r}^{-1}\left[\delta(b t) *\left(\underset{i=1}{*} T_{s_{i}} \rho_{i, t}\right)\right]
$$

where each $\rho_{i, t}$ is of the form given in (35).

\section{H-sets}

We turn now to some applications of the preceding theory. For their proper context, we refer the reader to $[7 ; 9 ; 18$, Chaps. IX, XII; and 1 , Chaps. XII, XIV]. In the 1920 s, Rajchman introduced the following generalization of Cantor's middle-thirds sets. 
Definition. A Borel set $E \subset \mathbb{T}$ is called an $\boldsymbol{H}$-set if there exist a sequence $\left\{\boldsymbol{m}_{j}\right\}_{j=1}^{\infty} \subset \mathbb{N}$ tending to $\infty$ and a non-empty open set $I \subset \mathbb{T}$ such that for every $x \in E$ and all $j, m_{j} x \notin I$.

Cantor's middle-thirds set is the set $\left\{x: \forall j \geq 0 \quad 3^{j} x \notin\right] \frac{1}{3}, \frac{2}{3}[\}$. The connection of $H$-sets to our preceding discussion is given by the following observation.

Proposition 7. Let $\mu \in M(T)$ be such that whenever (3) holds for a sequence $m_{j} \rightarrow \infty$, supp $\sigma_{x}=\mathbb{\mu} \mu$-a.e. Then $\mu E=0$ for all $H$-sets $E$.

Proof. Let $E$ be an $H$-set. Let $m_{j} \rightarrow \infty$ and $I$ be a non-empty open set such that $m_{j} x \notin I$ for $x \in E$. By choosing a subsequence of $\left\{m_{j}\right\}$ if necessary, we may assume that there is a $\sigma_{x}$ such that (3) holds and that $\left\{m_{j} x\right\} \sim \sigma_{x} \mu$-a.e. If $x \in E$, then clearly supp $\sigma_{x} \subset \mathbb{T} \backslash I$, whence supp $\sigma_{x} \neq \mathbb{T}$. The hypothesis implies, then, that $\mu(E)=0$.

We established in [7] and [9] that hyperlacunary Riesz products,

$$
\mu=\prod_{k \geq 0}\left(1+\operatorname{Re}\left\{\alpha_{k} e\left(n_{k} x\right)\right\}\right), n_{k+1} / n_{k} \rightarrow \infty,\left|\alpha_{k}\right| \leq 1,
$$

annihilate all $H$-sets; if we choose $\alpha_{k} \rightarrow 0$, then these are examples of measures whose Fourier-Stieltjes coefficients do not vanish at infinity but which annihilate all $H$-sets nevertheless. This disproved a conjecture of Rajchman. New counterexamples are given by the following theorem.

THEOREM 8. Let $\mu$ satisfy the hypotheses of Theorem 1. Then $\mu$ annihilates all $\mathrm{H}$-sets if and only if supp $\mu=\mathbb{T}$.

Note that if $\mu \neq \lambda$, then by $q$-invariance, $\hat{\mu}$ does not vanish at $\infty$.

Proof. The following facts are easily verified: if $\omega, \omega^{\prime}$ are positive measures with $\operatorname{supp} \omega=\mathbb{T}$ and $r \in \mathbb{Z}$, then $\operatorname{supp} T_{r} \omega=\operatorname{supp} T_{r}^{-1} \omega=\operatorname{supp}\left(\omega * \omega^{\prime}\right)=\mathbb{T}$. Therefore the measures $\sigma_{x}$ of (7) have full support if $\mu$ does, and consequently $\mu$ annihilates all $H$-sets.

The converse is trivial. Indeed, if $\mu$ is any $q$-invariant measure whose support misses a non-empty open set $I$, then by $q$-invariance, supp $\mu$ also misses $T_{q}^{-j} I$ for all $j \geq 0$. That is, $\mu$ is supported on the $H$-set $\left\{x: \forall j \quad q^{j} x \notin I\right\}$.

The following extension would be very interesting.

QUESTION. If $\mu$ is a q-invariant q-mixing probability measure of full support, does $\mu$ annihilate all $\mathrm{H}$-sets?

Of course, Theorems 3 and 5 and the discussion of $\S 4$ permit the statement of several theorems similar to Theorem 8 . We shall restrict ourselves to the two main classes of examples, Riesz products and Bernoulli convolutions.

THEOREM 9. Let $\mu$ be a Riesz product as in (29) (thus, $Q_{k}\left|Q_{k+1}{ }^{\prime},\right| d_{k} \mid=\mathrm{O}(1)$ ). Then $\mu$ annihilates all $H$-sets.

Proof. $\$ 4$ shows that $\sigma_{t}$ is a trigonometric polynomial, which certainly has full support, or is formed from Riesz products. But it is well-known that Riesz products 
have full support. (The proof is simple: if

define

$$
\rho=\prod_{k \doteq 0}\left(1+\operatorname{Re}\left\{\beta_{k} e\left(l_{k} t\right)\right\}\right),
$$

$$
P_{K}=\prod_{k=0}^{K}\left(1+\operatorname{Re}\left\{\beta_{k} e\left(l_{k} t\right)\right\}\right) \quad \text { and } \quad \rho_{K}=\prod_{k>K}\left(1+\operatorname{Re}\left\{\beta_{k} e\left(l_{k} t\right)\right\}\right) .
$$

Thus $\rho=P_{K} \cdot \rho_{K}$. If $\rho(I)=0$ for some open set $I$, then $\rho_{K}(I)=0$ since $P_{K}$ has at most finitely many zeros and $\rho_{K}$ is continuous. But since $\rho_{K} \rightarrow \lambda$ weak*, it follows that $\lambda I=0$.)

The same ideas apply to generalized Riesz products, of course. It would be very interesting to know whether all Riesz products annihilate all $H$-sets. Indeed, this question was the original motivation for the present work.

ThEOREM 10. Let $\mu$ be a Bernoulli convolution

$$
\mu=\underset{k \geq 1}{*} \sum_{i=0}^{\left|q_{k}\right|-1} p_{i, k} \delta\left(i Q_{k}^{-1}\right),
$$

where $\left|q_{k}\right| \geq 2$, sup $\left|q_{k}\right|<\infty$,

and for all $|q| \geq 2$ and all $i \in[0,|q|-1]$,

$$
Q_{k}=q_{1} q_{2} \cdots q_{k}, \sum_{i=0}^{q_{k}-1} p_{i, k}=1,
$$

$$
\liminf _{k \rightarrow \infty}\left\{p_{i, k}: q_{k}=q\right\}>0 \text {. }
$$

Then $\mu$ annihilates all $H$-sets.

Proof. It was shown that Theorem 3 is applicable; we only have to show that the weak* limit points of $\left\{T_{Q_{n}} \mu\right\}$ have full support. Let $T_{Q_{n_{i}}} \mu \rightarrow \nu$ weak* ${ }^{*}$. We may assume the existence of the following limits for all $k \geq 1$ :

$$
\tilde{q}_{k}=\lim _{j \rightarrow \infty} q_{n_{j}+k}, \quad \tilde{p}_{i, k}=\lim _{j \rightarrow \infty} p_{i, n_{j}+k} \quad\left(0 \leq i \leq\left|\tilde{q}_{k}\right|-1\right) .
$$

If $\tilde{Q}_{k}=\tilde{q}_{1} \cdots \tilde{q}_{k}$, we see that

$$
\nu=\underset{k \geqslant 1}{*} \sum_{i=0}^{\left|\tilde{q}_{k}\right|-1} \tilde{p}_{i, k} \delta\left(i \tilde{Q}_{k}^{-1}\right)
$$

Since $\tilde{p}_{i, k}>0$ by hypothesis, $\operatorname{supp} \nu=\mathbb{T}$.

It turns out that the converse of Proposition 7 holds as well. We first establish the following lemma.

LEMMA 11 [7]. Let $\mu$ be a positive measure on a measurable space $X$ without atoms of infinite measure. Let $E_{n}$ be measurable sets, $1_{n}$ the characteristic functions of $E_{n}$, and

$$
E=\left\{t: \lim _{N \rightarrow \infty} \frac{1}{N} \sum_{n=1}^{N} 1_{n}(t)=1\right\}
$$

be the set of points lying in almost all the $E_{n}$. Then

$$
\mu E \leq \sup _{\left\{n_{k}\right\}} \mu\left(\bigcap_{k=1}^{\infty} E_{n_{k}}\right),
$$


where $\left\{n_{k}\right\}$ runs over all sequences with $n_{k} \rightarrow \infty$. In particular, if $\mu\left(\bigcap_{k=1}^{\infty} E_{n_{k}}\right)=0$ for all $\left\{\boldsymbol{n}_{k}\right\}$, then $\mu E=0$.

Proof. By restricting $\mu$ to a subset of $E$ of finite measure, if necessary, it suffices to assume that $\mu$ is a probability measure concentrated on $E$. It follows that

$$
1=\int_{X} \lim _{N \rightarrow \infty} \frac{1}{N} \sum_{n=1}^{N} 1_{n}(t) d \mu(t)=\lim _{N \rightarrow \infty} \frac{1}{N} \sum_{n=1}^{N} \mu E_{n} .
$$

Given $\varepsilon>0$, there exists, therefore, a sequence $n_{k} \rightarrow \infty$ such that $\mu E_{n_{k}}>1-\varepsilon 2^{-k}$. Since $\mu\left(\bigcap_{k=1}^{\infty} E_{n_{k}}\right)>1-\varepsilon$, the lemma follows.

Definition [7]. A Borel set $E \subset \mathbb{T}$ is called an asymptotic $H$-set if there exists a sequence $m_{j} \rightarrow \infty$ and a non-empty open set $I \subset \mathbb{T}$ such that for $x \in E$,

$$
\lim _{j \rightarrow \infty} \frac{1}{J} \operatorname{card}\left\{j \leq J: m_{j} x \notin I\right\}=1 \text {. }
$$

Corollary 12 [7]. A measure annihilates all asymptotic $H$-sets [resp., those based on any subsequence of $\left\{m_{j}\right\}$ and $I$ ] if and only if it annihilates all $H$-sets [resp., those based on any subsequence of $\left\{\boldsymbol{m}_{j}\right\}$ and $\left.I\right]$.

Proof. This follows immediately from Lemma 11 applied to the sets

$$
E_{j}=\left\{x: m_{j} x \notin I\right\} .
$$

We are now able to give the following version of Proposition 7 and its converse.

THEOREM 13. Let $\mu \in M(\mathbb{T})$ and $m_{j} \rightarrow \infty$ be such that (3) holds. Then supp $\sigma_{x}=\mathbb{T}$ $\mu$-a.e. if and only if $\mu$ annihilates all $H$-sets based on any subsequence of $\left\{m_{j}\right\}$.

Proof. One direction was shown in the proof of Proposition 7. For the other, suppose that $\operatorname{supp} \sigma_{x} \neq \mathbb{T}$ on a set of positive $|\mu|$-measure. We may assume that $\left\{m_{j} x\right\} \sim \sigma_{x}$ $\mu$-a.e. Then there is a set $F^{\prime}$ of positive measure and an $\eta>0$ such that supp $\sigma_{x}$ misses some arc of length $\eta$ for every $x \in F^{\prime}$, whence there is a set $F$ of positive measure and a fixed arc $I^{\prime}$ of length $\eta / 2$ such that supp $\sigma_{x} \cap I^{\prime}=\varnothing$ for all $x \in F$. Let $I$ be a non-empty open arc whose closure is contained in the interior of $I^{\prime}$. Then

$$
\lim _{J \rightarrow \infty} \frac{1}{J} \operatorname{card}\left(j \leq J: m_{j} x \in I\right\}=0 \quad \text { for } x \in F,
$$

whence $F$ is an asymptotic $H$-set based on $\left\{m_{j}\right\}$. By Corollary 12 , there is a subsequence $\left\{m_{j}^{\prime}\right\}$ of $\left\{m_{j}\right\}$ such that the $H$-set $\left\{x: \forall j m_{j}^{\prime} x \notin I\right\}$ has positive $|\mu|$-measure.

Consider now the Cantor-Lebesgue measure

$$
\mu=\underset{k \geq 1}{*}\left[\frac{1}{2} \delta(0)+\frac{1}{2} \delta\left(2 \cdot 3^{-k}\right)\right]
$$

supported on the Cantor middle-thirds set. (We have chosen àn invariant $\mu$ for simplicity, not for any essential reason.) One expects intuitively that the only $H$-sets not annihilated by $\mu$ are those based on sequences sufficiently similar to $\left\{3^{j}\right\}$. This is true: 
ThEOREM 14. Let

$$
\mu=\underset{k \geqq 1}{*}\left[\frac{1}{2} \delta(0)+\frac{1}{2} \delta\left(2 \cdot 3^{-k}\right)\right]
$$

be the Cantor-Lebesgue measure. If $E$ is an $H$-set of positive $\mu$-measure corresponding to a sequence $\left\{m_{j}\right\}$, then every subsequence $\left\{m_{j}^{\prime}\right\}$ of $\left\{m_{j}\right\}$ has a further subsequence $\left\{m_{j}^{\prime \prime}\right\}$ of the form

$$
r m_{j}^{\prime \prime}=s 3^{n_{j}+b} \quad\left(r, s \in \mathbb{N}^{+}, b \in \mathbb{Z}, n_{j} \rightarrow \infty\right) .
$$

Conversely, if $\left\{m_{j}\right\}$ is of the form (36), then there is an $H$-set of positive $\mu$-measure corresponding to a subsequence of $\left\{\boldsymbol{m}_{j}\right\}$.

Proof. If $E \subset\left\{x: \forall j m_{j} x \notin I\right\}$ is an $H$-set of positive measure and $\left\{m_{j}^{\prime}\right\} \subset\left\{m_{j}\right\}$, then $\left\{x: \forall j m_{j}^{\prime} x \notin I\right\}$ is also of positive measure since it contains $E$. Thus, to prove the first half of the theorem, it suffices to prove only that $\left\{m_{j}\right\}$ has a subsequence $\left\{m_{j}^{\prime \prime}\right\}$ of the form (36).

Now let $\left\{m_{j}^{\prime \prime \prime}\right\}$ be a subsequence of $\left\{m_{j}\right\}$ such that $e\left(-k m_{j}^{\prime \prime \prime} x\right) \rightarrow \hat{\sigma}_{x}(k)$ weak* in $L^{\infty}(\mu)$. By (13), we know that

where

$$
\sigma_{x}=T_{r}^{-1}\left[\delta(b \zeta(x)) *\left(\underset{i=1}{\stackrel{*}{*}} T_{s_{i}} \nu\right)\right] \mu \text {-a.e., }
$$

$$
\nu=\underset{k \geq 1}{*}\left[\frac{1}{2} \delta(0)+\frac{1}{2} \delta\left(3^{-k}\right)\right],
$$

with $l=1$ if and only if $\left\{m_{j}^{\prime \prime \prime}\right\}$ has a further subsequence $\left\{m_{j}^{\prime \prime}\right\}$ of the form (36). Thus, by Theorem 13, the present theorem reduces to showing that $l \neq 1$ if and only if supp $\sigma_{x}=\mathbb{T} \mu$-a.e.

Suppose first that $l=1$. Then $\operatorname{supp} T_{s_{1}} \nu=\left\{s_{1} x: x \in \operatorname{supp} \nu\right\}$. Since $\operatorname{supp} \nu$ is a nowhere dense set, so is supp $T_{s_{1}} \nu$, and so, therefore, is supp $\sigma_{x}$.

Conversely, suppose that $l>1$. We shall show that $\operatorname{supp}\left(T_{s_{1}} \nu * T_{s_{2}} \nu\right)=\pi$. Since $\nu$ is 3 -invariant, we may assume that $3 \nmid s_{1} s_{2}$. Now

so that

$$
T_{s} \nu=\underset{k \geq 1}{*}\left[\frac{1}{2} \delta(0)+\frac{1}{2} \delta\left(s 3^{-k}\right)\right]
$$

$$
\rho \stackrel{\text { def }}{=} T_{s_{1}} \nu * T_{s_{2}} \nu=\underset{k \geq 1}{*}\left[\frac{1}{4} \delta(0)+\frac{1}{4} \delta\left(s_{1} 3^{-k}\right)+\frac{1}{4} \delta\left(s_{2} 3^{-k}\right)+\frac{1}{4} \delta\left(\left(s_{1}+s_{2}\right) 3^{-k}\right)\right]=\xi_{K} * \omega_{K}
$$

for any $K \geq 1$, where $\xi_{K}$ is the discrete measure formed by the convolution of the first $K$ terms and $\omega_{K}$ is the probability measure formed by the remainder. Now supp $\rho=\mathbb{T} \Leftrightarrow \rho I>0$ for every arc $I$ of the form

$$
\begin{gathered}
I=\left[A-\left(\left|s_{1}\right|+\left|s_{2}\right|\right) 3^{-K}, A+\left(\left|s_{1}\right|+\left|s_{2}\right|\right) 3^{-K}\right], \\
A=\sum_{k=1}^{K} a_{k} 3^{-k}, \quad a_{k} \in\{0,1,2\}, \quad K \geq 1 .
\end{gathered}
$$

Given such an arc, we can choose $\varepsilon_{K}, \varepsilon_{K}^{\prime} \in\{0,1\}$ such that

$$
\varepsilon_{K} s_{1}+\varepsilon_{K}^{\prime} s_{2} \equiv a_{K} \quad(\bmod 3)
$$

since $3 \nmid s_{1} s_{2}$. We may then choose $\varepsilon_{K-1}, \varepsilon_{K-1}^{\prime} \in\{0,1\}$ such that

$$
\left(\varepsilon_{K-1} s_{1}+\varepsilon_{K-1}^{\prime} s_{2}\right) 3+\left(\varepsilon_{K} s_{1}+\varepsilon_{K}^{\prime} s_{2}\right) \equiv a_{K-1} 3+a_{K}\left(\bmod 3^{2}\right),
$$


and so on, until we have chosen $\varepsilon_{k}, \varepsilon_{k}^{\prime} \in\{0,1\}(1 \leq k \leq K)$ such that

$$
\sum_{k=1}^{K}\left(\varepsilon_{k} s_{1}+\varepsilon_{k}^{\prime} s_{2}\right) 3^{K-k} \equiv \sum_{k=1}^{K} a_{k} 3^{K-k}\left(\bmod 3^{K}\right),
$$

which is the same as

$$
\sum_{k=1}^{K}\left(\varepsilon_{k} s_{1}+\varepsilon_{k}^{\prime} s_{2}\right) 3^{-k} \equiv A \quad(\bmod 1)
$$

Therefore $\xi_{K}(\{A\}) \geq(1 / 4)^{K}$; since supp $\omega_{K} \subset\left[-\left(\left|s_{1}\right|+\left|s_{2}\right|\right) 3^{-K},\left(\left|s_{1}\right|+\left|s_{2}\right|\right) 3^{-K}\right]$, it follows that $\rho I \geq 4^{-K}$.

We now present a similar example which will be useful in a moment.

LEMMA 15. Let

$$
\pi=\underset{\substack{k \geq 1 \\ k \notin \mathcal{N}}}{*}\left[\frac{1}{2} \delta(0)+\frac{1}{2} \delta\left(2^{-k}\right)\right],
$$

where $\mathcal{N}=\left\{n_{j}\right\}_{j \geq 1}$ is a sequence such that $n_{j+1}-n_{j} \rightarrow \infty$. If $\left\{m_{j}\right\}$ is a sequence which corresponds to an $\mathrm{H}$-set of positive $\pi$-measure,then there is a subsequence $\left\{m_{j}^{\prime}\right\}$ of the form

$$
r m_{j}^{\prime}=s 2^{n_{j}^{\prime-1}+b} \quad\left(r, s \in \mathbb{N}^{+}, b \in \mathbb{Z}, n_{j}^{\prime} \in \mathcal{N}\right) .
$$

Proof. We may assume that $e\left(-k m_{j} x\right) \rightarrow \hat{\sigma}_{x}(k)$ weak ${ }^{*}$ in $L^{\infty}(\pi)$. If we interpret $\pi$ as containing the terms $1 \cdot \delta(0)+0 \cdot \delta\left(2^{-k}\right)$ for $k \in \mathcal{N}$, then Theorem 3 is applicable by the discussion which followed that theorem. Thus,

$$
\sigma_{x}=T_{r}^{-1}\left[\delta(b x) *\left(\underset{i=1}{\stackrel{*}{*}} T_{s_{i}} \nu_{i}\right)\right] \pi \text {-a.e., }
$$

where each $\nu_{i}$ is a weak* limit point of $\left\{T_{2^{k}} \pi\right\}$. Suppose that $T_{2^{k}} \pi \rightarrow \nu$ weak* If $\left|k_{j}-\mathcal{N}\right|$ is unbounded, then it is easy to see that $\nu=\lambda$. If $\left|k_{j}-\mathcal{N}\right|$ is bounded, then without loss of generality, $k_{j}=n_{j}^{\prime}+d$, where $n_{j}^{\prime} \in \mathcal{N}$. If $d \geq 0$, then $\nu=\lambda$; if $d<0$, then

$$
\nu=\underset{\substack{k \geq 1 \\ k \neq-d}}{*}\left[\frac{1}{2} \delta(0)+\frac{1}{2} \delta\left(2^{-k}\right)\right]
$$

Since every $\nu_{i}$ is of this form and supp $\sigma_{x} \neq \mathbb{T}$ for a set of positive $\pi$-measure, it follows that $l=1$ and that for some $\left\{m_{j}^{\prime}\right\} \subset\left\{m_{j}\right\}, r m_{j}^{\prime}=s 2^{k_{j}}+b$ with $k_{j}=n_{j}^{\prime}+d$, $n_{j}^{\prime} \in \mathcal{N}$, and $d \leq-1$. Therefore $\left(r 2^{-d-1}\right) m_{j}^{\prime}=s 2^{n_{j}^{\prime}-1}+\left(b 2^{-d-1}\right)$, which is the desired form.

The following generalization of $\boldsymbol{H}$-sets was introduced by Pjateckil-Šapiro [14; 18, Chap. XII, § 11; 1, Chap. XIV, § 15; 9].

Definition. Let $m \in \mathbb{Z}^{+}$. If

$$
V=\left(v^{(1)}, \ldots, v^{(m)}\right) \in \mathbb{Z}^{m}, \Lambda=\left(l_{1}, \ldots, l_{m}\right) \in \mathbb{Z}^{m}
$$

and $x \in \mathbb{T}$, we write $V \cdot \Lambda=\sum_{i=1}^{m} v^{(i)} l_{i}$ and $V x=\left(v^{(1)} x, \ldots, v^{(m)} x\right)$. A sequence $\left\{V_{k}\right\}_{1}^{\infty} \subset\left(\mathbb{Z}^{+}\right)^{m}$ of $m$-tuples of positive integers is called quasi-independent if for each fixed $\Lambda \in \mathbb{Z}^{m}, \Lambda$ not the 0 -vector, we have $\left|V_{k} \cdot \Lambda\right| \rightarrow \infty$ as $k \rightarrow \infty$. A Borel set $E \subset \mathbb{T}$ is called an $H^{(m)}$-set if there exist a quasi-independent sequence $\left\{V_{k}\right\} \subset\left(\mathbb{Z}^{+}\right)^{m}$ and a non-empty open set $I \subset \mathbb{T}^{m}$ such that for all $x \in E$ and all $k, V_{k} x \notin I$. 
In [7] and [9], we asked whether for each $m \geq 1$, there is a measure supported on an $H^{(m+1)}$-set which annihilates all $H^{(m)}$-sets, in other words, whether $H^{(m+1)}$ is 'much larger' than $H^{(m)}$. Here we show that the answer is 'yes' for $m=1$.

THEOREM 16. Let $\pi$ be the measure in Lemma 15,

$$
\rho=\underset{j \geq 1}{*}\left[\frac{1}{3} \delta(0)+\frac{1}{3} \delta\left(2^{-n_{2 j-1}}\right)+\frac{1}{3} \delta\left(2^{-n_{2 j}}\right)\right], \quad \text { and } \mu=\pi * \rho .
$$

Then $\mu$ is supported on an $H^{(2)}$-set and annihilates all $H$-sets.

Proof. Indeed, $\mu$ is supported on the 'canonical' $H^{(2)}$-set

$$
\left\{x: \forall j\left(2^{n_{2 j-1}-1} x, 2^{n_{2 j}-1} x\right) \notin\right]^{\frac{1}{2}}, 1[\times] \frac{1}{2}, 1[\} .
$$

Suppose that $E$ were an $H$-set corresponding to a sequence $\left\{m_{j}\right\}$ with $\mu E>0$. Since $\mu E=\int_{\pi} \pi(E-t) d \rho(t)$, it would follow that $\pi(E-t)>0$ for some $t$. But $E-t$ is an $H$-set corresponding to a subsequence $\left\{\boldsymbol{m}_{j}^{\prime}\right\} \subset\left\{\boldsymbol{m}_{j}\right\}$ (if $\left\{\boldsymbol{m}_{j}^{\prime}\right\}$ is chosen so that $\left\{m_{j}^{\prime} t\right\}$ is almost constant, then $\left\{m_{j}^{\prime} x\right\}$ is not dense for $\left.x \in E-t\right)$. Lemma 15 shows that for a further subsequence $\left\{m_{j}^{\prime \prime}\right\} \subset\left\{m_{j}^{\prime}\right\}$, we have

$$
r m_{j}^{\prime \prime}=s 2^{n_{j}^{\prime}-1}+b \quad \text { with } n_{j}^{\prime} \in \mathcal{N} \text {. }
$$

Let $e\left(-k m_{j}^{\prime \prime} x\right) \rightarrow \hat{\sigma}_{x}(k)$ and $e\left(-k 2^{n_{j}^{\prime}-1} x\right) \rightarrow \hat{\tau}_{x}(k)$ weak* in $L^{\infty}(\mu)$. It is not hard to calculate that

$$
\tau_{x}=\left[\frac{2}{3} \delta(0)+\frac{1}{3} \delta\left(2^{-1}\right)\right] *\left[\underset{k \geq 2}{*}\left(\frac{1}{2} \delta(0)+\frac{1}{2} \delta\left(2^{-k}\right)\right)\right] \mu \text {-a.e. }
$$

(This can also be calculated by convolving the weak* limits in $L^{\infty}(\pi)$ and $L^{\infty}(\rho)$; see [10].) Of course, supp $\tau_{x}=\pi$; since $\sigma_{x}=T_{r}^{-1}\left[\delta(b x) * T_{s} \tau_{x}\right]$, we also have supp $\sigma_{x}=\mathbb{T}$, which completes the proof by contradicting Theorem 13.

Acknowledgements. This research was conducted largely while the author was at the Université de Paris (Orsay), to whom I am grateful for their hospitality. I would also like to thank Jean-François Méla, Martine Queffelec, Bernard Host, and François Parreau for encouraging me to present my work in their seminar at the Université de Paris (Villetaneuse).

\section{REFERENCES}

[1] Nina K. Bari. A Treatise on Trigonometric Series. Translated from the Russian by M. F. Mullins. Volumes I, II. New York: Macmillan, 1964.

[2] J. R. Blum \& Bernard Epstein. On the Fourier-Stieltjes coefficients of Cantor-type distributions. Israel J. Math. 17 (1974), 35-45.

[3] I. P. Cornfeld, S. V. Fomin \& Ya. G. Sinai. Ergodic Theory. Translated from the Russian by A. B. Sossinskii. New York: Springer, 1982.

[4] Colin C. Graham \& O. Carruth McGehee. Essays in Commutative Harmonic Analysis. New York: Springer, 1979.

[5] Yitzhak Katznelson. An Introduction to Harmonic Analysis. Second corrected edition. New York: Dover, 1976.

[6] Russell Lyons. Measure-theoretic quantifiers and Haar measure. Proc. Amer. Math. Soc. 86 (1982), 67-70; Erratum, Proc. Amer. Math. Soc. 91 (1984), 329-330.

[7] Russell Lyons. A Characterization of Measures whose Fourier-Stieltjes Transforms Vanish at Infinity. Dissertation, The University of Michigan, 1983. 
[8] Russell Lyons, Fourier-Stieltjes coefficients and asymptotic distribution modulo 1. Ann. of Math. 122 (1985), 155-170.

[9] Russell Lyons. The size of some classes of thin sets. Studia Math., 86 (1987), 59-78.

[10] Russell Lyons. The local structure of some measure-algebra homomorphisms. Preprint (1988).

[11] Yves Meyer. Les produits de Riesz sont des Bernoulli-shifts. (d'après Yitzhak Katznelson et Benjamin Weiss), unpublished.

[12] John C. Oxtoby. Ergodic sets. Bull. Amer. Math. Soc. 58 (1952), 116-136.

[13] B. Petit. Schémas de Bernoulli et $g$-mesures. C. R. Acad. Sc. Paris 280, série A (1975), 17-20.

[14] I. I. Pjatecki1-Šapiro. On the problem of uniqueness of expansion of a function in a trigonometric series, (in Russian). Moscov. Gos. Univ. Uč. Zap. 155, Mat. 5 (1952), 54-72. Supplement to the work 'On the problem...,' (in Russian), Moscov. Gos. Univ. Uč. Zap. 165, Mat. 7 (1954), 79-97.

[15] V. A. Rokhlin. Exact endomorphisms of a Lebesgue space. Izv. Akad. Nauk SSSR Ser. Mat. 25 (1961), 499-530 (Russian); English transl., Amer. Math. Soc. Transl. (2) 39 (1964), 1-36.

[16] Meir Smorodinsky. Ergodic Theory, Entropy. Berlin-Heidelberg-New York: Springer, 1971.

[17] Hermann Weyl. Über die Gleichverteilung von Zahlen mod. Eins. Math. Ann. 77 (1916), 313-352. Also in 'Gesammelte Abhandlungen', vol. 1, pp. 563-599, Berlin: Springer, 1968.

[18] Antoni Zygmund. Trigonometric Series. Second edition, reprinted. Volumes I, II. Cambridge: Cambridge University Press, 1979. 